\title{
Solar Ultraviolet Bursts
}

\author{
Peter R. Young ${ }^{1,2,3}\left(\mathbb{D} \cdot\right.$ Hui Tian ${ }^{4} \cdot$ Hardi Peter $^{5}$ - Robert J. Rutten ${ }^{6,7,8}$ • \\ Chris J. Nelson ${ }^{9,10}$ • Zhenghua Huang ${ }^{11}$ - Brigitte Schmieder ${ }^{12}$ - Gregal J.M. Vissers ${ }^{13}$. \\ Shin Toriumi ${ }^{14}$ - Luc H.M. Rouppe van der Voort ${ }^{7,8}$ • Maria S. Madjarska ${ }^{5}$. \\ Sanja Danilovic ${ }^{13}$ - Arkadiusz Berlicki ${ }^{15,16}$ - L.P. Chitta ${ }^{5}$ Mark C.M. Cheung ${ }^{17}$. \\ Chad Madsen $^{18,19}$ • Kevin P. Reardon ${ }^{20,21}$ • Yukio Katsukawa ${ }^{14}$ - Petr Heinzel ${ }^{16}$
}

Received: 27 April 2018 / Accepted: 5 October 2018 / Published online: 6 November 2018

(C) The Author(s) 2018

\begin{abstract}
The term "ultraviolet (UV) burst" is introduced to describe small, intense, transient brightenings in ultraviolet images of solar active regions. We inventorize their properties and provide a definition based on image sequences in transition-region lines. Coronal signatures are rare, and most bursts are associated with small-scale, canceling oppositepolarity fields in the photosphere that occur in emerging flux regions, moving magnetic features in sunspot moats, and sunspot light bridges. We also compare UV bursts with similar transition-region phenomena found previously in solar ultraviolet spectrometry and with similar phenomena at optical wavelengths, in particular Ellerman bombs. Akin to the lat-
\end{abstract}

Electronic supplementary material The online version of this article

(https://doi.org/10.1007/s11214-018-0551-0) contains supplementary material, which is available to authorized users.

\footnotetext{
$凶$ P.R. Young

pyoung9@gmu.edu

1 College of Science, George Mason University, 4400 University Drive, Fairfax, VA 22030, USA

2 NASA Goddard Space Flight Center, Code 671, Greenbelt, MD 20771, USA

3 Northumbria University, Newcastle Upon Tyne NE1 8ST, UK

4 School of Earth and Space Sciences, Peking University, Beijing 100871, China

5 Max Planck Institute for Solar System Research, Justus-von-Liebig-Weg 3, 37077, Göttingen, Germany

6 Lingezicht Astrophysics, 't Oosteneind 9, 4158 CA Deil, The Netherlands

7 Institute of Theoretical Astrophysics, University of Oslo, PO Box 1029 Blindern, 0315 Oslo, Norway

8 Rosseland Centre for Solar Physics, PO Box 1029 Blindern, 0315 Oslo, Norway

9 School of Mathematics and Statistics, University of Sheffield, Hicks Building, Hounsfield Road, Sheffield S3 7RH, UK

10 Astrophysics Research Centre, School of Mathematics and Physics, Queen's University, Belfast, BT7 1NN Northern Ireland, UK

11 Shandong Provincial Key Laboratory of Optical Astronomy and Solar-Terrestrial Environment, Institute of Space Sciences, Shandong University, Weihai, 264209 Shandong, China
} 
ter, UV bursts are probably small-scale magnetic reconnection events occurring in the low atmosphere, at photospheric and/or chromospheric heights. Their intense emission in lines with optically thin formation gives unique diagnostic opportunities for studying the physics of magnetic reconnection in the low solar atmosphere. This paper is a review report from an International Space Science Institute team that met in 2016-2017.

Keywords Sun: atmosphere $\cdot$ Sun: activity $\cdot$ Sun: UV radiation $\cdot$ Sun: transition region

\section{Introduction}

The Sun's chromosphere and corona were identified well before the space age. The two orders of magnitude difference between their temperatures suggested there is a thin, abrupt transition in which the temperature rises very rapidly with height (e.g., Mariska 1992). Despite its thinness this "transition region" (TR) was found to emit strongly in most resonance lines in the ultraviolet (UV) between 500 and $1600 \AA$. The lines in this region are generally called "TR lines" and specific phenomena observed in them "TR phenomena". The lines are usually assigned characteristic formation temperatures in the $20-800 \mathrm{kK}$ range that are derived by assuming optically-thin ionization equilibrium. In this paper we will identify ions with their temperatures of maximum ionization $\left(T_{\max }\right)$, but readers should be aware that for highly dynamic phenomena the emission may be formed in plasma out of equilibrium and the effective temperature of formation may differ from $T_{\max }$. This has been demonstrated in models of coronal loops by Noci et al. (1989) and in models of the IRIS Si IV and O IV emission lines by Doyle et al. (2013) and Olluri et al. (2013). Note that the high densities inferred for UV bursts (e.g., Peter et al. 2014) may limit these effects as discussed by Young et al. (2018).

Imaging in specific UV lines is rarely achieved due to technology restrictions, so that so far most TR phenomena were classified through their spectral UV features only, with particular emphasis on large Doppler shifts, excessive broadening (larger than the thermal Doppler width defined by the $T_{\max }$ value), and complex profiles.

12 LESIA, Observatoire de Paris, PSL Research University, CNRS, Sorbonne Universités, UPMC Univ. Paris 06, Univ. Paris Diderot, Sorbonne Paris Cité, 5 place Jules Janssen, 92195 Meudon, France

13 Institute for Solar Physics, Department of Astronomy, AlbaNova University Centre, Stockholm University, 10691 Stockholm, Sweden

National Astronomical Observatory of Japan, 2-21-1 Osawa, Mitaka, Tokyo 181-8588, Japan

Astronomical Institute, University of Wrocław, Kopernika 11, 51-622 Wrocław, Poland

Astronomical Institute, Academy of Sciences of the Czech Republic, 25165 Ondrejov, Czech Republic USA 
The Interface Region Imaging Spectrometer (IRIS, De Pontieu et al. 2014) is the newest UV spectrometer to observe solar TR lines. The telescope feeds a slit spectrometer, providing spectra at unprecedented spatial and spectral resolution (Sect. 3). In addition, a Slit-Jaw Imager (SJI) yields simultaneous images in four bandpasses. The one at $1400 \AA$ is of particular interest as it is often dominated by two Si IV lines, making IRIS the first mission to routinely obtain high-resolution images in TR lines (Appendix A). When combined with simultaneous spectroscopy of these lines, the environment of TR phenomena, including their history, can be identified, facilitating interpretation of their UV signatures in terms of other solar fine structures found with other diagnostics.

In most energetic events, the two Si IV line profiles have factor-two peak and profile ratios indicating optically thin line formation (Peter et al. 2014; Kim et al. 2015; Vissers et al. 2015). This is a valuable asset as their intensities therefore represent a more direct measure of energy release than the complex, optically-thick, source function mapping of lines such as the $\mathrm{C}$ II and $\mathrm{Mg}$ II $\mathrm{h} \& \mathrm{k}$ resonance lines sampled by IRIS. Their dominance of the IRIS $1400 \AA$ slit-jaw passband over the continuum contribution wherever there is substantial heating delivers direct, high-cadence, wide-field imaging of such events (example in panel b of Fig. 1).

An early discovery from IRIS (Peter et al. 2014) was a type of very intense, compact brightening in active regions with highly complex Si IV profiles called a bomb ("IRIS bombs", IB) in view of striking similarities with Ellerman bombs (EB; Ellerman 1917). This discovery inspired the first author to convene an International Space Science Institute (ISSI) International Team to study these bombs and other intense UV brightenings detected in IRIS spectra and images. The team met twice, in 2016 January and 2017 March, with the subject "Solar UV bursts-a new insight to magnetic reconnection". The term UV burst was chosen to denote the full scale of IRIS brightening events. Their discussions included the following major issues: How are UV bursts related to EBs and the magnetic field? Can underlying patterns be recognised in different types? Can complex burst line profiles be reproduced with numerical simulation codes?

The present article summarizes these discussions and the conclusions reached at these meetings, and also provides observational criteria to classify UV bursts in other IRIS data.

Section 2 presents our definition of a UV burst. Section 3 summarizes transient TR phenomena observed with previous UV instrumentation. Section 4 presents a summary of observational UV burst results obtained so far from IRIS, while Sect. 5 describes efforts in modeling these. A final summary is given in Sect. 6 .

\section{UV Burst Definition}

Most solar phenomena are identified from their morphology in images or image sequences. For example, relatively stable structures such as prominences, sunspots and coronal loops are easily identified on individual images, while transient features such as flares and jets are identified from fast-cadence image sequences. Do UV bursts possess distinct signatures that can be readily employed for their identification?

Figure 1 shows near-simultaneous snapshots from 10-minute active-region image sequences obtained with IRIS and the Solar Dynamics Observatory (SDO). A movie version covering the whole sequence in the same format accompanies this manuscript. The IRIS $1400 \AA$ slit-jaw image (SJI) in panel (b) shows a number of compact bright grains (black in the images) that are much brighter than their surroundings. Viewing the full sequence in the 

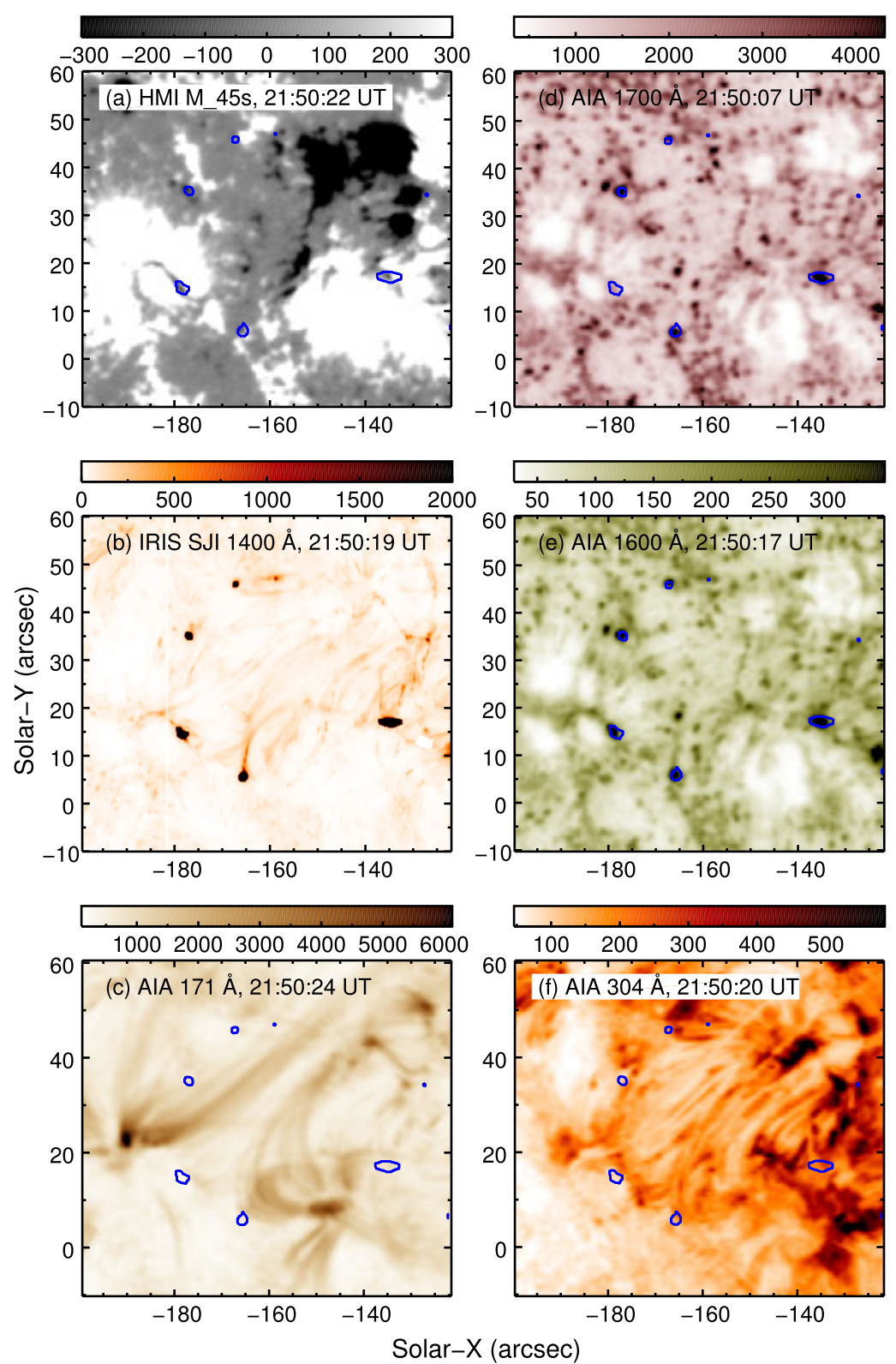

Fig. 1 Images illustrating UV bursts from a 10-minute 18-second cadence IRIS sequence showing part of active region AR 11875 on October 22, 2013 at solar $(X, Y)=(-162.25)$ during 21:45-21:55 UT. Panel (a): line-of-sight (LOS) HMI magnetogram scaled between $\pm 300 \mathrm{G}$. Panel (b): IRIS SJI $1400 \AA$ A. Panels (c)-(f): AIA $171 \AA$, $1700 \AA, 1600 \AA$ and $304 \AA$ images. Plotted quantity for panels (b)-(f) is intensity in DN s ${ }^{-1}$. The blue contours superimposed on panels (a) and (c)-(f) outline the brightest bursts in panel (b), at a level of $1000 \mathrm{DN} \mathrm{s}^{-1}$ 
movie shows that their brightness flickers, that some are present for the entire duration, and that others are short-lived. We call such features UV bursts.

Figure 1 also shows the co-temporal line-of-sight (LOS) magnetic field from the Helioseismic and Magnetic Imager (HMI; Scherrer et al. 2012) on board SDO, and UV images from SDO's Atmospheric Imaging Assembly (AIA; Lemen et al. 2012). The AIA $171 \AA$ filter is dominated by Fe IX $\lambda 171.1$, formed at $0.8 \mathrm{MK}$. The $304 \AA$ filter is dominated by the He II Ly $\alpha$-like resonance line and is formed around $80 \mathrm{kK}$. The AIA $1600 \AA$ and $1700 \AA$ channels sample the upper photosphere and lower chromosphere, with small magnetic concentrations appearing bright from their evacuation. If atmospheric heating is sufficiently large then the C IV $\lambda \lambda 1548,1550 \AA$ resonance lines can dominate over the continuum in the $1600 \AA$ channel, and this is often the case for UV bursts hence the stronger contrast compared to the $1700 \AA$ channel. Note that the $1600 \AA$ channel has a significantly broader filter width than the SJI $1400 \AA$ channel and the continuum is more intense, thus the SJI channel is much more effective for TR imaging.

Our name "burst" denotes the brief duration of the brightening events. As such it is a standard astronomy term, as in "gamma-ray bursts" and "fast radio bursts". This name has also been used before to describe solar TR phenomena (Sect. 3.4).

We append "UV" to burst instead of "transition region" to keep closer to observation and because "region" in the latter is somewhat a misnomer. In the actual solar atmosphere every radial column must naturally contain a steep temperature rise, but its height varies tremendously both spatially and temporally and does not define a layer or shell as in traditional one-dimensional equilibrium models such as the classical ones of Vernazza et al. (1981). A sharper and physically sounder description is to define the chromosphere-corona transition at any location and instant as the height where hydrogen reaches $50 \%$ ionization-generally a non-equilibrium quantity making up a highly warped, dynamic, and history-dependent surface. There are probably no phenomena restricted solely to this instantaneous transition surface; more likely heating events with increasing ionization come from below (such as the bursts here) whereas cooling events with increasing recombination come from above (such as coronal rain). The "TR" phenomena reported in the older literature must represent spectral UV signatures of wider-origin events. Our goal is to re-classify them into the latter by exploiting IRIS' UV imaging capability.

Thus, we prefer the term "UV bursts". The UV wavelength range 465 to $1550 \AA$ (bounded by Ne VII $\lambda 465$ and C IV $\lambda 1550$ ) is indeed dominated by chromosphere-corona transition lines, although there are also some chromospheric and coronal lines in this range.

Figure 1 and its movie version give a quick impression of UV bursts; now we define them more formally. They are identified in image sequences sampling ultraviolet passbands that are dominated by a chromosphere-corona transition emission line, and have the following properties:

1. Compactness. Core brightenings $\lesssim 2^{\prime \prime}$ in size, typically $\leq 1^{\prime \prime}$. A burst may appear with extended structure (jet, fibril, loop) connected to it, but these are typically less bright. The burst itself may also appear spatially extended into one direction ("flame"), but remains $\lesssim 2$ ". Two or more bursts may appear close together but remain associated with the same magnetic feature in the photosphere;

2. Duration. Bursts can have lifetimes ranging from tens of seconds to over an hour. For long-lived ones the intensity is not constant, but may flicker by about a factor two on timescales around a minute. At high angular resolution such bursts can also appear as a sequence of intermittent, repetitive flarings, possibly with a migrating footpoint;

3. Intensity. The burst is significantly brighter than the surroundings in the pertinent UV passband. For Fig. 1(b) the blue contours correspond to a factor 24 above the image 
median value. Peak SJI $1400 \AA$ intensities of individual spatial pixels of the brighter events can reach factors of 100-1000 higher than the median.

4. Motion. UV bursts show only small proper motions, typically $\leq 10 \mathrm{~km} \mathrm{~s}^{-1}$. That is, they generally track dynamics of photospheric magnetic features and their interactions, rather than traveling-front or wave motions along extended structures such as loops;

5. UV bursts are not directly related to flares. This condition distinguishes UV bursts from the compact, intense kernels that appear along flare ribbons and otherwise look similar.

We do not recommend setting a specific intensity threshold for UV bursts, as there is no physical reason to exclude a wide spectrum of events with different energies and temperatures defining the signal in the pertinent wavelength bands. Instead, a dataset-specific threshold suits better to let the observer obtain a manageable number of events for study, for example by specifying a factor above the median intensity value (such as 24 for panel $b$ of Fig. 1), or a factor $\mathrm{k} \sigma$ above the median value with $\sigma$ the standard deviation of the intensity over an image ( $k \approx 7$ for the $1400 \AA$ data in Fig. 1$)$. Also note that integrated intensity over the burst area may define a more appropriate threshold than the intensity of the brightest spatial pixel in the burst.

The above criteria also include most IBs identified in the literature, but with the important difference that the latter are defined through their spectroscopic signatures discussed in Sect. 4. At least one type of event, the narrow line bursts of Hou et al. (2016), do not satisfy the IB criteria while satisfying the UV burst criteria. Therefore we envision IBs to be a subclass of UV bursts, although probably the dominant one.

Section 4 describes key results from the IRIS papers in more detail; we summarize these here and we refer the reader to Sect. 4 for further details and references to the literature:

- Si IV line profiles of UV bursts mostly have a complex shape that can include multiple peaks, large excess broadening beyond the thermal width (at temperature $T_{\max }$ ), and very extended wings. They often indicate the presence of bi-modal jets; in slanted limbward viewing these may also be mapped into profile differences along different LOSs to the burst. However, some events show simple, Gaussian-shaped profiles not much wider than the thermal width;

- UV bursts generally overlie or are directly adjacent to small magnetic features in the photosphere that interact (usually cancelation following convergent proper motion) with similar or larger opposite-polarity features;

- Most UV bursts do not show significant co-spatial brightening in the AIA $171 \AA$ A channel (dominated by Fe IX, formed at $0.8 \mathrm{MK}$ ), nor in the other coronal AIA channels, and only rarely in the He II $304 \AA$ passband;

- EBs are more common than UV bursts, with around 10-20\% of EBs having a burst signature while $30-60 \%$ of bursts show an EB signature.

These should be considered properties of UV bursts, but are not part of their definition above.

The general consensus from the literature and our ISSI team is that UV bursts are smallscale magnetic reconnection events, and that their complex Si IV line profiles possibly display dynamics associated with a current sheet; for example fast-moving, dense plasmoids, ${ }^{1}$

\footnotetext{
${ }^{1}$ The term plasmoid applies to magnetic islands that appear in models of current sheets subject to the tearing instability and also to observed features in the Earth's magnetotail, where the magnetic field can be measured in situ. For remote-sensing solar observations, the term is often applied to small-scale brightenings, but it is not possible to determine if these are the same as the current sheet/magnetotail plasmoids without magnetic field measurements.
} 
Table 1 Properties of TR instruments

\begin{tabular}{|c|c|c|c|c|}
\hline \multirow[t]{2}{*}{ Instrument } & \multirow[t]{2}{*}{ Years } & \multirow{2}{*}{$\begin{array}{l}\text { Wavelength } \\
\text { Range/Å }\end{array}$} & \multicolumn{2}{|l|}{ Resolution } \\
\hline & & & Spatial/arcsec & Spectral/Å \\
\hline Skylab S082B & 1973-1974 & $970-1940$ & 2 & 0.06 \\
\hline HRTS & 1975-1992 & $1170-1700$ & 1 & 0.1 \\
\hline SMM/UVSP & 1980-1989 & $1070-3600$ & 4 & 0.05 \\
\hline SOHO/SUMER & 1996-2017 & $500-1600$ & $2-3$ & 0.04 \\
\hline $\mathrm{SOHO} / \mathrm{CDS}$ & 1996-2013 & $308-381,515-630$ & $6-10$ & $0.3-0.5$ \\
\hline Hinode/EIS & 2006-present & $170-212,246-292$ & $3-4$ & 0.06 \\
\hline IRIS & 2013-present & $1332-1358,1389-1407$ & 0.3 & 0.026 \\
\hline
\end{tabular}

or turbulence induced in surrounding plasma by reconnection outflow jets. The reconnection occurs somewhere in the upper photosphere-to-upper chromosphere regime, rather deep in the atmosphere. The particular location and also the line of sight to the reconnection site may be responsible for differences in burst signatures such as variations in Si IV profiles.

Our UV burst definition requires transition region imaging capability, which was either not available with instruments prior to IRIS or infrequently used (Appendix A). If a slit spectrometer is raster-stepping to build an image scan, only infrequent snapshots of the burst will be obtained, and just one if it is short-lived. If there is only one snapshot the evolution and proper motion cannot be analyzed. However, it may be possible to estimate at least the latter from supporting data, for example, magnetograms (HMI or better) or magneticconcentration monitoring in AIA 1600 and $1700 \AA$ images. Also, if the burst intensity happens to be low at the time(s) of snapshot sampling it may fail high-intensity threshold criteria. Therefore raster mode data tend to underestimate the number of UV bursts, but some may get identified properly.

In sit-and-stare mode following mean solar rotation $\left(10^{\prime \prime} / \mathrm{hr}\right.$ at disk center) the time evolution can be accurately monitored as long as the burst remains within the slit, but if the burst has low intensity during this sampling period it may not be flagged at all. Also, the spatial extent of a burst cannot be established with fixed-slit data, which may lead to erroneous feature classification.

\section{Previous Event Types}

TR event types are generally quite different to coronal events such as coronal hole plumes, active region loops and coronal bright points. These have typical spatial extents of 10's of arcseconds and lifetimes from hours to days, compared to sizes of a few arcseconds or less, and lifetimes of minutes for TR events. In addition the availability of coronal EUV and $\mathrm{X}$-ray imaging means coronal features are typically identified from spatial morphology. The various TR event types have almost exclusively been identified through UV spectrometry, and so definitions to some extent depend on the capabilities of the instruments, in particular their spatial resolution, spectral resolution and coverage. Table 1 summarizes the characteristics of the instruments treated in this section. Note that due to multiple configurations or changes in time, the listed parameters are not complete; the listed values are typical for TR measurements by these instruments. The table shows that IRIS has better spatial and spectral resolutions compared to other instruments by factors of three and two, respectively. 
Table 2 Key UV lines sampling the chromosphere-corona transition

\begin{tabular}{llll}
\hline Ion & $\log \left(T_{\max } / \mathrm{K}\right)$ & Wavelength $(\AA)$ & Instruments \\
\hline Si IV & 4.90 & $1393.78,1402.77$ & HRTS, UVSP, SUMER, IRIS \\
C IV & 5.05 & $1548.19,1550.77$ & HRTS, UVSP, SUMER \\
O IV & 5.15 & 1401.16 & HRTS, UVSP, SUMER, IRIS \\
N v & 5.30 & $1238.82,1242.80$ & HRTS, SUMER \\
O v & 5.40 & 629.73 & CDS, SUMER $(\times 2)$ \\
& & 192.90 & EIS \\
\hline
\end{tabular}

The first report of strongly broadened TR lines was Brueckner et al. (1976) who presented spectra of TR instabilities observed as very broad features in the C IV $\lambda \lambda 1548,1550$ doublet observed with the Skylab S082B instrument. This spectrometer had effectively no spatial resolution along the slit, so that further analysis awaited flights of the High Resolution Telescope Spectrograph (HRTS) rocket experiment. HRTS was the first solar instrument to observe the TR at high spectral and spatial resolution. It was flown ten times on rockets between 1975 and 1997 and once as part of the Spacelab 2 payload in $1985 .^{2}$

Long-term monitoring at high spatial resolution of the TR began with the UltraViolet Spectrometer and Polarimeter (UVSP) on board the Solar Maximum Mission during 1984 to 1989. The Solar Ultraviolet Measurements of Emitted Radiation (SUMER) and Coronal Diagnostic Spectrometer (CDS) on board the Solar and Heliospheric Observatory (SOHO) were the next major spectrometers with TR coverage, observing for almost 20 years since 1996. The EUV Imaging Spectrometer (EIS) on board the Hinode spacecraft was launched in 2006 and, although mostly focused on the corona, it observes a number of upper TR emission lines. Finally, IRIS was launched in 2013. It observes UV lines with significantly higher spatial and spectral resolution than previous instruments, and adds highly-valuable slitjaw imaging.

We now discuss different types of TR features that were identified with these instruments in the light of our UV bursts. The emission lines that were most commonly used are given in Table 2, with $T_{\max }$ values derived from the CHIANTI database (Dere et al. 1997; Del Zanna et al. 2015; Young et al. 2016). We suggest that especially the so-called SMM bursts (Sect. 3.4) and possibly also active region blinkers (Sect. 3.5) directly correspond to UV bursts, based on the emission measure comparison in Appendix B.

\subsection{Jets}

In recent solar physics the term jet is usually applied to transient, elongated and extending structures that are seen in emission in image sequences. The term surge pre-dates the use of jet in the solar physics literature (e.g., Zirin 1966) and is generally used to describe similar structures that are mostly seen in absorption in chromospheric lines (particularly $\mathrm{H} \alpha$ ).

The lack of TR imaging capability prior to IRIS means that the analogs of coronal or chromospheric jets could not be directly identified. Instead TR spectra have been reported of jets identified from other imaging data. For example, Schmieder et al. (1983) studied $\mathrm{SMM} / \mathrm{UVSP}$ spectra of a surge identified from $\mathrm{H} \alpha$ images, and they measured blueshifts and line broadening in the C IV lines. Similarly, Madjarska (2011) captured SOHO/SUMER

\footnotetext{
${ }^{2} \mathrm{~A}$ brief history of HRTS is available at http://wwwsolar.nrl.navy.mil/hrts_hist5.html.
} 
spectra of a coronal jet identified from EUV and X-ray imaging data, and found blueshifts of up to $300 \mathrm{~km} \mathrm{~s}^{-1}$ in several TR lines.

The term jet was applied specifically to a TR spectral feature by Brueckner and Bartoe (1983) who identified intense emission in Si IV and C IV lines that always appeared blueshifted and extended out to $400 \mathrm{~km} \mathrm{~s}^{-1}$. Subsequent work showed that these features are quite rare, however (Dere 1994).

Since the launch of IRIS, jets can now be identified directly from TR imaging data, and examples include the quiet Sun network jets of Tian et al. (2014b) and the active region jets of Cheung et al. (2015).

A jet may correspond to a UV burst if there is an intense brightening at the jet base that passes the criteria of Sect. 2. The column of the jet, where large velocities are expected, generally would not pass the criteria because the emission is typically weak and extends over several or more arcseconds.

\subsection{Penumbral Microjets and Sunspot Dots}

Penumbral microjets were first described by Katsukawa et al. (2007) based on Hinode Solar Optical Telescope filtergram images centered on the Ca II $\mathrm{H}$ line. They are small jets occurring within a sunspot's penumbra with lengths typically 1 to $4 \mathrm{Mm}$ and lifetimes $\leq 1$ minute. Many can be identified at any one time within a penumbra. They often have a different orientation than the adjacent penumbral filaments. Cospatial observations with IRIS and the Swedish Solar Telescope (SST) in Ca II (Vissers et al. 2015) demonstrated that these features also appear in Si IV as a compact brightening at the top of the jet with an intensity enhancement of around five compared to the surroundings.

Tian et al. (2014a) found brightness features in IRIS SJI $1330 \AA$ and $1400 \AA$ images that also occur mainly in sunspot penumbrae, which they termed bright dots. They have a progressively weaker signal in lower atmosphere layers, with most events having no signature in $\mathrm{H} \alpha$ (Deng et al. 2016). Tian et al. (2014a) suggested that bright dots have a connection to the Ca II penumbral microjets which was largely confirmed by Vissers et al. (2015), but Samanta et al. (2017) identified a distinct class of bright dots without Ca II signature. Penumbral bright dots were also found in $193 \AA$ images from the Hi-C rocket flight (Alpert et al. 2016), which were not considered to be coronal features but contributed by cooler TR emission lines in the $193 \AA$ passband. Tiwari et al. (2016) found signatures of larger penumbral jets in the AIA coronal filters, and suggested a different formation mechanism for these compared to the standard microjets. Bright dots in a sunspot umbra were identified by Chitta et al. (2016); they lie at the footpoints of coronal loops.

We note that sunspot bright dots are excluded from our UV burst definition due to their relatively weak intensity enhancement in SJI $1400 \AA$ images and their large proper motions of $10-40 \mathrm{~km} \mathrm{~s}^{-1}$ (Tian et al. 2014a). However, since penumbral microjets and some bright dots are found at locations of convective intrusion into strong field, the physics of these events may have similarities to the bursts found in umbral light bridges (Sect. 4.3).

\subsection{Explosive Events}

This name was introduced by Dere et al. (1989) for events found with the third HRTS rocket flight in 1979; earlier they were named "turbulent events" by Brueckner and Bartoe (1983). They were identified as large broadening of C IV $\lambda 1548$, on one side of the line only or on both sides but with asymmetrical wings. Wherever both wings were enhanced there was often a spatial separation along the slit of up to $2^{\prime \prime}$. The maximum Doppler shift was not found 
to vary with the position of the events on the solar disk, suggesting that the corresponding mass motions are isotropic. This led the authors to regard the events as explosions, hence the name "explosive event".

Most of the HRTS-era explosive events were measured in quiet areas, with perhaps the most significant active region event reported by Brueckner et al. (1988) with the HRTS on Spacelab 2 in 1985. This was reported to be only five times more intense than nearby active region plage. Although difference in spatial resolution must be accounted for when comparing intensities of compact features from different instruments, this relatively low enhancement suggests that the various HRTS flights did not observe anything directly equivalent to the IBs of Peter et al. (2014) since those are characterized by much larger intensity enhancements of the Si IV lines.

There were a number explosive event studies from SUMER data (see Huang et al. 2014, for a summary of results), but almost all were focused on quiet Sun or coronal holes as active regions on the disk were rarely observed due to concerns over instrument degradation. One exception is the event presented by Brekke et al. (2000) that showed high velocity features in ions ranging from N IV to Ne VIII $\left(\log T_{\max }(\mathrm{K})=5.2-5.8\right)$ although, based on the images presented in the paper, it did not show the strong intensity enhancement characteristic of IBs.

The only IRIS paper that has presented a quiet Sun explosive event is that of Huang et al. (2014). The event occurred at the boundary of an equatorial extension of a polar coronal hole, and it exhibited strongly broadened Si IV lines. The high spatial resolution of the SJI $1330 \AA$ images allowed small jets to be seen, which are the first direct evidence of the jets inferred from the high Doppler broadening in spectral data. Gupta and Tripathi (2015) and Huang et al. (2017) studied active region events that they referred to as explosive events, but we consider these to be UV bursts and they are discussed in the following sections.

\subsection{Bursts}

The use of the word "burst" with regard to TR phenomena dates back to the Skylab period, with Emslie and Noyes (1978) and Widing (1982) referring to impulsive solar bursts. These showed sudden increases in UV emission-line intensities, with two events associated with flares and two not. Of direct relevance to the IRIS observations is the study by Hayes and Shine (1987) who reported observations from the SMM/UVSP instrument with the Si IV $\lambda 1402.77$ and O IV $\lambda 1401.16$ lines that are also observed by IRIS. They defined bursts as events for which the Si IV $\lambda 1402.77$ intensity increased by a factor two in one minute. The line width and velocity were not part of this definition. The emission measure analysis presented in Appendix B suggests that they observed equivalents of IBs.

We also note that Innes et al. (1997) used the term burst to refer to a contiguous train of explosive events, lasting for up to 30 minutes. Studies of some IRIS bursts suggest similar behavior (e.g., Vissers et al. 2015; Gupta and Tripathi 2015).

\subsection{Blinkers}

The term blinker was introduced by Harrison (1997) to describe small-scale brightenings seen in quiet-Sun areas with the Coronal Diagnostic Spectrometer (CDS) on board SOHO. More detailed studies followed by Bewsher et al. (2002) and Parnell et al. (2002), who applied an automatic detection method. They were identified from peaks in light curves for individual emission lines by requiring that the peak be a factor $P$ times the noise level of nearby light-curve minima. In addition, this criterion must be satisfied for $N$ neighboring 
spatial pixels. Ranges of $P$ and $N$ values were investigated, with $P=5$ and $N=3$ chosen for statistical analysis. The OV $\lambda 629.7$ line was the strongest TR line observed by CDS and was the main reference line used in these studies.

Unlike SUMER, the CDS instrument routinely observed active regions on the solar disk so, although it lacked the spectral resolution required to study broadened profiles of explosive events or IBs, it was well capable of detecting large UV-line enhancements. An early study by Young and Mason (1997) found two intense UV brightenings in the core of a small, recently-emerged active region that were factors of $\approx 50$ brighter than the average quiet Sun. The study of active region blinkers of Parnell et al. (2002) yielded a wide spectrum of blinker events, with the most intense being around a factor 100 times stronger than average activeregion emission. The emission measure analysis of Appendix B shows that $\mathrm{O} v$ blinkers are about an order of magnitude weaker than UV bursts in Si IV, but we consider them to be consistent with the UV burst definition. Young (2004) studied a handful of the most intense active region blinker events and found densities as high as $10^{11}-10^{12} \mathrm{~cm}^{-3}$, although only one event exhibited significantly enhanced non-thermal broadening.

The Hinode/EIS instrument mostly observes coronal emission lines, but a number of lines with $T_{\max }$ values from 0.1 to $0.7 \mathrm{MK}$ are also observed, as highlighted by Young et al. (2007), although they are significantly weaker than the strong TR lines observed by CDS, SUMER and IRIS. An intense UV brightening was identified in Young et al. (2007) and considered analogous to CDS active-region blinkers. Despite this, we are aware of only one other comparable brightening reported in the literature, that of Guglielmino et al. (2010). A systematic search for these brightenings in existing EIS data-sets and additional joint EIS-IRIS observing campaigns are required to determine if there is any correspondence between the EIS events and IBs.

\subsection{Ellerman Bombs}

Ellerman (1917) referred to these as "solar hydrogen bombs", but Ellerman bomb (EB) is the preferred name today. EBs are photospheric features with sizes $\leq 2$ " and lifetimes of several minutes that have traditionally been identified only in complex emerging active regions, but similar phenomena occur in sunspot moats and were also recently found in quiet-Sun areas (Rouppe van der Voort et al. 2016; Nelson et al. 2017). They are defined by their signature in the $\mathrm{H} \alpha$ line, consisting of strong enhancements in the red and blue wings. Crucially, these wing intensity enhancements are significantly larger than for the much more common quiescent magnetic flux concentrations that cause wing brightening by the hot-wall effect, a phenomenon different from EBs but sometimes causing confusion (Rutten et al. 2013). Several studies (Vissers et al. 2013; Nelson et al. 2013; Vissers et al. 2015; Nelson et al. 2015; Reid et al. 2015, 2016) suggest that enhancement of about $50 \%$ above the mean at $\pm 1 \AA$ from $\mathrm{H} \alpha$ center is a good criterion to separate EBs from flux concentrations. When observed towards the limb at high angular resolution (at least of $0.2^{\prime \prime}$ ) EBs are found to have distinctive flame-like appearance in images obtained in the wing of $\mathrm{H} \alpha$ (Hashimoto et al. 2010; Watanabe et al. 2011). Recent 3D MHD simulations have been able to reproduce this tell-tale characteristic remarkably well (Danilovic 2017; Hansteen et al. 2017).

EBs occur where bi-polar small-scale magnetic fields move together and cancel, typically at locations of emerging flux in active regions as stipulated by Ellerman (1917), but also at moving magnetic features (MMF) in moats around sunspots. They are interpreted as magnetic reconnection events occurring below $1000 \mathrm{~km}$ height (Georgoulis et al. 2002; Pariat et al. 2004; Watanabe et al. 2011; Vissers et al. 2013). Emerging-flux EBs were discussed in the review article of Schmieder et al. (2015). 
EBs are sometimes found at the base of $\mathrm{H} \alpha$ surges-large jet-like structures seen in absorption in filtergrams taken in a H $\alpha$ wing-but less often than claimed by Roy (1973). He also reported surges from EB-like brightenings in light bridges. These different magnetic environments are further discussed in Sect. 4.3.

\section{UV Burst Results from IRIS}

Most IRIS studies in this context focused on IRIS bombs (IBs) that were introduced by Peter et al. (2014) who named these for similarities with Ellerman bombs. Other terms have also been used, such as hot explosions (Kim et al. 2015), explosive events (Gupta and Tripathi 2015; Huang et al. 2017), compact brightenings (Grubecka et al. 2016) and flaring activeregion filaments (Vissers et al. 2015). In the present discussion we assume that they are all UV bursts under the definition in Sect. 2 above.

To illustrate the properties of IBs and UV bursts in this section, we show four examples from the literature in Fig. 2. Bursts (a) and (b) correspond to bomb events 4 and 1, respectively, of Peter et al. (2014); burst (c) is the event studied by Gupta and Tripathi (2015); burst (d) is an event from the data-set studied by Toriumi et al. (2015b). For each burst we show the spectral image for Si IV $\lambda 1402.77$ (leftmost panels), with the nearest-in-time IRIS slit-jaw (SJI) $1400 \AA$ image (middle panels) and the corresponding LOS magnetograms from HMI (rightmost panels). The images were spatially aligned using the AIA $1600 \AA$ channel images, which also reveal the bursts (yellow contours in right panels). We emphasize the high intensity of the Si IV lines by displaying values of the lines' maximum specific intensities in the left panels. For comparison, an average quiet Sun Si IV $\lambda 1402.77$ profile has a peak specific intensity of about $800 \mathrm{erg} \mathrm{cm}^{-2} \mathrm{~s}^{-1} \mathrm{sr}^{-1} \AA^{-1}$ and a full-width at half-maximum of $0.2 \AA$. Average active region line profiles typically have peaks in the 5000-20000 erg cm ${ }^{-2} \mathrm{~s}^{-1} \mathrm{sr}^{-1} \AA^{-1}$ range.

The IBs were identified by Peter et al. (2014) from a $140 \times 170 \operatorname{arcsec}^{2}$ IRIS raster scan taken on 2013 September 24 during 11:44-12:04 UT. The authors did not explicitly state criteria that defined IBs, but they formulated the four properties given below. We consider criteria (2) to be too strict, as noted in the following text.

1. the Si IV lines are very wide with wings reaching out to $\approx 200 \mathrm{~km} \mathrm{~s}^{-1}$ separation from line center;

2. the Si IV intensities are enhanced by a factor $\sim 1000$ compared to the surrounding active region;

3. narrow atomic and single-ionized absorption lines are superimposed as absorption blends on the IRIS lines, including the Si IV lines;

4. the ratio of Si IV $\lambda 1402.77$ to O IV $\lambda 1401.16$ becomes much larger than usual for areas where both appear, suggesting very high densities $\gtrsim 10^{13} \mathrm{~cm}^{-3}$.

Whereas very wide lines are typical of explosive events (Sect. 3.3), properties (2)-(4) were not previously reported for such events. Yan et al. (2015) demonstrated that recognizing property (3) requires the high spectral resolution of IRIS.

The line width criterion (1) is required to ensure the line is broad enough to display the narrow absorption blends. Note that it does not refer to the full-width at half-maximum of the line, as is often used for describing Gaussian-shaped lines. The strongest absorption line (Ni II $\lambda 1393.33$ ) lies at $-91 \mathrm{~km} \mathrm{~s}^{-1}$ from the center of Si IV $\lambda 1393.76$ - see the red profiles in the left panels of Fig. 2(a)-(c). 


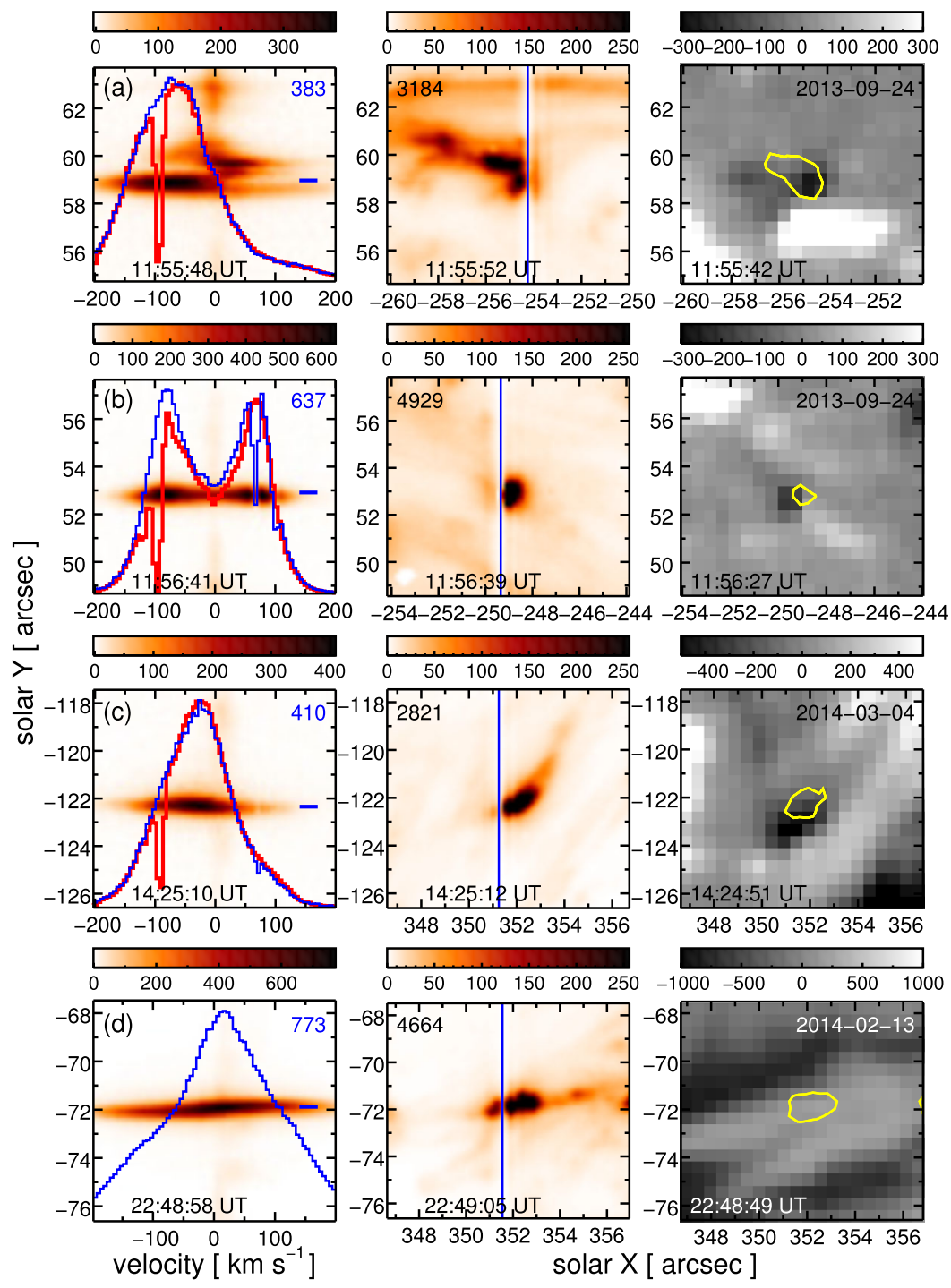

Fig. 2 Four examples of intense bursts. The leftmost panels show IRIS spectrograms for the spectral band $\pm 200 \mathrm{~km} \mathrm{~s}^{-1}$ around Si IV $\lambda 1402.77$; the center panels show SJI $1400 \AA$ images; the rightmost panels show HMI LOS magnetograms. The blue and red curves superimposed in the first column show the 1D spectra for Si IV $\lambda 1402.77$ and $\lambda 1393.76$, respectively, at the $Y$-pixel specified by the short blue line at right, with $\lambda 1393.76$ scaled down by a factor two. The yellow contours in the third column are taken from corresponding AIA $1600 \AA$ images. The blue vertical lines in the center panels show the position of the IRIS slit. The blue numbers in the left panels give the maximum specific intensity in units $10^{3} \mathrm{erg} \mathrm{cm}^{-2} \mathrm{~s}^{-1} \mathrm{sr}^{-1} \AA^{-1}$ for the $1 \mathrm{D}$ spectra; the color bar gives the same units. The numbers in the center panels give the maximum image intensity in DN. The observation dates are shown at top-right of the rightmost panels

The IB intensity criterion above applies only to the most extreme events, and many of the IBs in the literature do not reach this intensity. Also, since IBs are defined from their spectroscopic signature, the intensity at the instant the IRIS slit crosses the event is unlikely to 

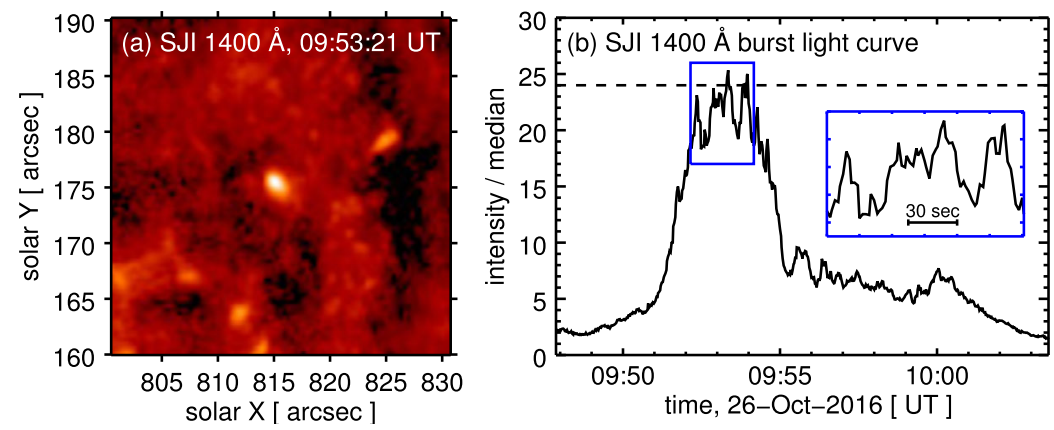

Fig. 3 Rapid variability in a UV burst. Panel (a) shows an IRIS SJI $1400 \AA$ image of a burst (image center) with a logarithmic intensity scale. Panel (b) shows the intensity variability of this burst from an area of $7 \times 8$ pixels centered on the feature and expressed relative to the median of the larger area displayed in panel (a). The blue box indicates the region shown in greater detail in the inset plot. The horizontal dashed line shows the intensity level used for the IRIS intensity contours in Fig. 1

be the highest value. This is particularly relevant when trying to associate IBs with features identified from imaging data (e.g., Zhao et al. 2017). Gupta and Tripathi (2015) demonstrated that IB criteria (1), (3) and (4) are valid for most of the lifetime of the event they studied, which was captured with a sit-and-stare observation. If only a single snapshot had been obtained with a raster scan, then the event may have failed criterion (2). Assessing the feature intensity by measurement on IRIS SJI $1400 \AA$ images is to be preferred. It has not yet been demonstrated whether events exist that satisfy IB criteria (1), (3) and (4) but have only a weak intensity enhancement, say a factor 5 to 10 .

To put the IB intensities in context, we first use an IRIS quiet Sun dataset from 2013 October 3 04:20-04:37 UT to measure an average quiet Sun Si IV $\lambda 1402.77$ intensity of $160 \mathrm{erg} \mathrm{cm}^{-2} \mathrm{~s}^{-1} \mathrm{sr}^{-1}$. The average intensity ${ }^{3}$ of the active region studied by Peter et al. (2014) was $2800 \mathrm{erg} \mathrm{cm}^{-2} \mathrm{~s}^{-1} \mathrm{sr}^{-1}$, and the intensity of the brightest spatial pixel of IB number 1 of Peter et al. (2014) was $5.2 \times 10^{5} \mathrm{erg} \mathrm{cm}^{-2} \mathrm{~s}^{-1} \mathrm{sr}^{-1}$. We caution that the sensitivity of IRIS has degraded significantly since launch, with version 4 of the radiometric calibration (as implemented through the IDL procedure iris_get_response) showing a decrease in sensitivity of a factor 4 at $1402.8 \AA$ between 2013 August and 2017 August. It is therefore recommended to always quote intensities in calibrated units rather than data number $(\mathrm{DN})$ units in order that bursts at different times can be compared.

One feature of UV bursts is the flickering of their intensities over time. Light curves constructed from the IRIS/SJI $1400 \AA$ images are shown by Peter et al. (2014), Vissers et al. (2015) and Kim et al. (2015) and reveal that the intensity evolution of a UV burst consists of many individual peaks that are often close to the SJI temporal resolution. The cadence per SJI channel is usually lower than for the spectrometer exposures due to cycling between different SJI channels. The fastest possible cadence of SJI imaging is 1.7 seconds, and we have identified one data-set beginning at 09:08 UT on 2016 October 16 that has this cadence for the SJI $1400 \AA$ channel and contains bursts. Figure 3(a) shows an image of one burst from this dataset, captured at the peak brightness. Panel (b) shows the light curve of the burst and the inset plot shows a close up of the variability near the peak intensity. Individual spikes at the sequence cadence can be identified, which suggests that the full variability of the burst is

\footnotetext{
${ }^{3}$ Peter et al. (2014) did not give intensities in calibrated units; the values quoted in this sentence were obtained by the lead author of the present study.
} 

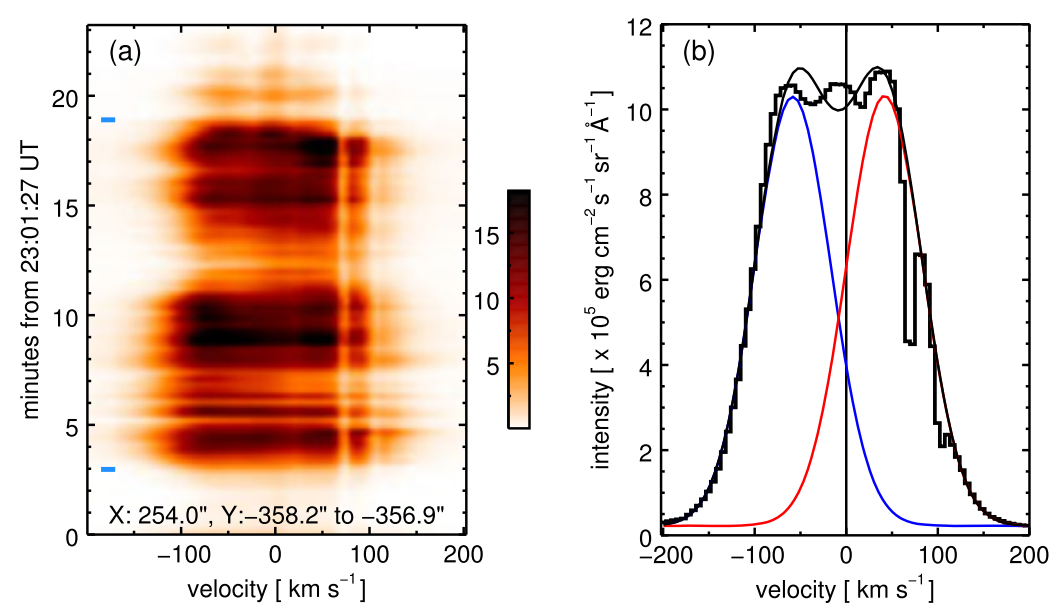

Fig. 4 Panel (a) shows the evolution of the line profile of Si IV $\lambda 1402.8$ for a UV burst observed on 2014 October 24. The spatial coordinates of the burst are indicated and a reverse, linear intensity scaling is applied. Short horizontal blue lines identify the time range that was averaged to yield the line profile (thick black line) in panel (b). Two Gaussians were fit to the profile and are shown as red and blue lines; the thin black line shows the total fitted profile

not being captured at this cadence. However, we also note that the frame-to-frame intensity variation for this event is relatively small at around $10 \%$ or less.

Gupta and Tripathi (2015) presented sit-and-stare observations that captured a UV burst with a five second cadence. Figure 7 of this work shows that the Si IV emission line light curve exhibits strong variability on this timescale. These authors applied wavelet analysis to identify periods of 30 and 60-90 seconds in the C II and Si IV spectral lines. We note that flickering has previously been reported for EBs, for example by Pariat et al. (2007) and Watanabe et al. (2011).

\subsection{Emission Line Profiles}

The Si IV profiles of IBs presented in the literature show significant shape variations; Fig. 2 gives some flavor of this. The striking double-peaked profile found by Peter et al. (2014) and shown in Fig. $2 b$ is not common, but has been seen in other events, for example, Fig. 3 of Rouppe van der Voort et al. (2017) and Fig. 7 of Tian et al. (2016). More commonly the line has a dominant blue or red component (panels a and c of Fig. 2) or only a small Doppler shift of the centroid (Fig. 2d). Some profiles show Si IV wings extending to over $2.5 \AA\left(530 \mathrm{~km} \mathrm{~s}^{-1}\right)$ from line center (Fig. 16 of Vissers et al. 2015), and a common feature is a triangular profile shape, i.e., the profiles' sides are linear when plotted as wavelength vs. $\log$ (intensity) as opposed to the parabolic shape of Gaussian profiles. Examples are shown in Figs. 4 to 8 of Tian et al. (2016).

One possibility for the complex shapes of IB line profiles is that the IRIS exposures represent a time average of a set of simpler, but rapidly-varying line profiles. For example, one could imagine that individual plasmoids are being rapidly produced by the plasma, each with a characteristic large speed but in different directions. The sum of the line profiles would then yield a broad, complex spectral feature. The observations in the literature typically use exposure times of 2 to 8 seconds so the characteristic timescale for the evolution 

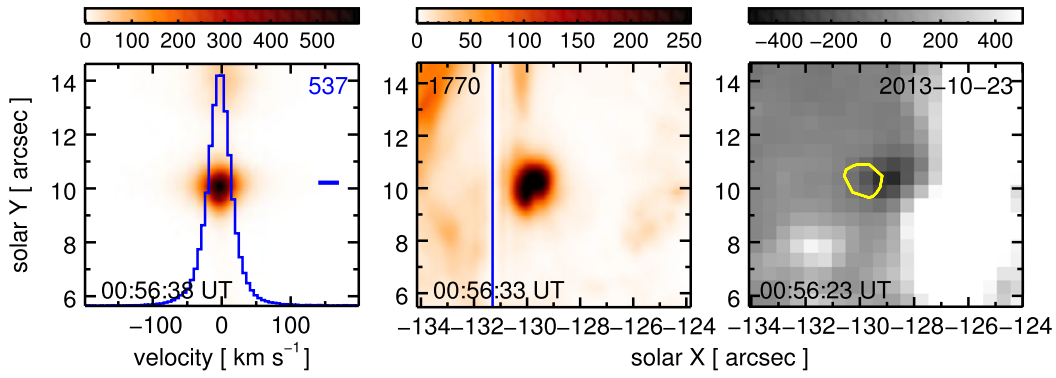

Fig. 5 An example of a narrow-line UV burst from an IRIS raster obtained between 00:41 and 00:51 UT on 2013 October 23. The format is the same as for Fig. 2

of individual line profiles would have to be a fraction of a second. Evidence against this comes from the widths of the emission lines in sit-and-stare sequences, which can remain approximately the same over timescales of minutes. An example is the evolution of the Si IV lines in the UV burst presented in Fig. 2 of Gupta and Tripathi (2015). Although the line widths vary significantly with time, they remain approximately constant for periods of about a minute (about 20 exposures). Another example is shown in Fig. 4 where the evolution of Si IV $\lambda 1402.8$ for a burst observed on 2014 October 24 at 23:02 UT is shown, and we see that the line width remains about $200 \mathrm{~km} \mathrm{~s}^{-1}$ for 15 minutes. This is difficult to explain unless the basic velocity structure of the plasma remains fairly stable over timescales of minutes.

The Si IV profiles often vary significantly across IBs along the slit, for example in Fig. 2 of Yan et al. (2015), Fig. 6 of Grubecka et al. (2016), and Fig. 5 of Chitta et al. (2017). In the latter case a MMF was studied and redshifts were found on the forward side of the MMF, blueshifts on the rearward side, which was interpreted as reconnection jets tilted with respect to the line of sight.

A defining feature of IBs is the presence of atomic or single-ionized blends superimposed on the broad Si IV emission lines. The deepest of the absorption lines is usually Ni II $\lambda 1393.33$, superimposed on Si IV $\lambda 1393.76$. Sometimes the latter is not returned in the IRIS telemetry stream, however, and absorption is less easy to identify in Si IV $\lambda 1402.77$ (compare profiles in the leftmost column of Fig. 2). When present, the narrow absorption dips have relatively small Doppler shifts (generally less than $10 \mathrm{~km} \mathrm{~s}^{-1}$ ) and betray the presence of relatively cool gas (hydrogen and helium predominantly neutral) along the line of sight of the burst that produces the wide profiles.

Occasionally absorption features near the rest velocities of the Si IV lines can be identified, and examples have been shown by Yan et al. (2015) and Vissers et al. (2015). We note that the latter paper suggested the absorption occurs in an overlying layer rather than due to the burst emission itself being optically thick.

In addition to the complex line profile patterns, bursts with very narrow, Gaussian-shaped Si IV line profiles have been reported by Hou et al. (2016). They were found above sunspots and the authors suggested a connection with sunspot plumes. The most intense event had an intensity of $3.5 \times 10^{5} \mathrm{erg} \mathrm{cm}^{-2} \mathrm{~s}^{-1} \mathrm{sr}^{-1}$ in the brightest spatial pixel, comparable to the IBs of Peter et al. (2014). These events thus appear to satisfy the UV burst criteria, but the narrow line profiles disqualifies them from being IBs. The ISSI team found other examples of intense, narrow-line bursts, and Fig. 5 shows an example from 2013 October 23, although this one is not above a sunspot. A key requirement is to distinguish narrow-line bursts from mini-flare ribbons (Sect. 4.5) and dynamic loops (Sect. 4.6), that may also show narrow line 
profiles. A wider survey of narrow-line bursts and a study of their underlying magnetic field structure will be worthwhile.

The complex UV burst profiles suggests that some details of the reconnection physics are being revealed in the UV lines; a first modeling effort was performed by Innes et al. (2015) who considered how plasmoids in a current sheet may lead to line profile shapes as observed, in particular triangular profiles. New high spatial resolution chromospheric images from the CHROMIS instrument (Rouppe van der Voort et al. 2017) show tiny brightenings of sizes close to $0.1^{\prime \prime}$ that are associated with a burst and may correspond to plasmoids. The authors also simulated Si IV emission using a 2.5D radiative MHD simulation and found that plasmoids could be responsible for the complex Si IV profiles if the line-of-sight passes through multiple plasmoids along the current sheet.

\subsection{Energy Estimates for UV Bursts}

In this section we give a rough estimate of the energy of a typical burst, using the example from 2014 October 24 shown in Fig. 4. We assume that the burst plasma is isothermal at the temperature of formation of Si IV $(80 \mathrm{kK})$. As noted in the previous section the Si IV profile remains approximately the same during the event lifetime (Fig. 4) and we average the $\lambda 1402.8$ line over the 16 minute period indicated in Fig. 4 a to yield the profile shown in panel b. For simplicity we fit this with two Gaussians drawn as red and blue lines on Fig. 4b. The fit does not reproduce the fine structure at the top of the profile, but the width and total intensity are accurately reproduced. The Gaussians have LOS velocities of -58 and $+42 \mathrm{~km} \mathrm{~s}^{-1}$, equal intensities and widths of $4.9 \times 10^{5} \mathrm{erg} \mathrm{cm}^{-2} \mathrm{~s}^{-1} \mathrm{sr}^{-1}$ and $0.46 \AA$, respectively. The latter corresponds to a non-thermal velocity of $59 \mathrm{~km} \mathrm{~s}^{-1}$ after subtracting the thermal and instrumental widths. The burst image drifted through the IRIS slit during the observation, and the full lifetime derived from SJI images was 36 minutes. The burst is about $1 \operatorname{arcsec}(725 \mathrm{~km})$ in size. The O IV $\lambda 1401.2$ emission line is very weak and we estimate a Si IV $\lambda 1402.8 / \mathrm{O}$ IV $\lambda 1401.2$ ratio of 317 , corresponding to an electron number density of $6.3 \times 10^{12} \mathrm{~cm}^{-3}$ using the results of Young et al. (2018).

Given the large Doppler velocities of the two line components and the small size of the burst, the event essentially blows itself apart in about 7 seconds (the travel time from the center of the burst to the edge). The burst can thus be considered to regenerate itself 309 times during the 36 minute lifetime. We refer to these as sub-bursts.

For each sub-burst, the energy inputs are as follows. The particles need to be heated from 10 to $80 \mathrm{kK}$; they are given an instantaneous kinetic energy corresponding to a bulk speed of $\approx 50 \mathrm{~km} \mathrm{~s}^{-1}$; they are given random non-thermal velocities of $59 \mathrm{~km} \mathrm{~s}^{-1}$; and heating is applied during the sub-burst lifetime to balance the radiative losses and maintain the burst's temperature.

The number of particles in the burst at any one time is, on average, given by $n=$ $2.3 E M_{V} / N_{\mathrm{e}}$, where the volume emission measure is defined in Appendix B. The average Si IV intensity is $9.8 \times 10^{5} \mathrm{erg} \mathrm{cm}^{-2} \mathrm{~s}^{-1} \mathrm{sr}^{-1}$, thus giving $E M_{V}=2.9 \times 10^{46} \mathrm{~cm}^{-3}$ and $n=1.1 \times 10^{34}$. If we assume these particles are heated from 10 to $80 \mathrm{kK}$, then the energy required for a sub-burst is $1.5 n k \Delta T=1.6 \times 10^{23} \mathrm{erg}$.

The combined bulk flow and random motions are given by $0.5 n m\left(v^{2}+\xi^{2}\right)$, where $m$ is the average mass of a particle taken here as 0.6 the mass of a proton. This gives a kinetic energy of $3.3 \times 10^{23} \mathrm{erg}$ for each sub-burst.

The energy loss rate due to radiation is given by $E M_{V} Q(T)$, where $Q(T)$ is the radiative loss function. At $80 \mathrm{kK}$ it takes the value of $4 \times 10^{-22} \mathrm{erg} \mathrm{cm}^{3} \mathrm{~s}^{-1}$ calculated using CHIANTI with photospheric abundances. For the 7 seconds lifetime of the sub-burst, the radiative 
losses are $8.1 \times 10^{25} \mathrm{erg}$, over two orders of magnitude larger than the thermal and kinetic energy terms,

Multiplying these energy estimates by the number of sub-bursts gives the total energy requirement of $2.5 \times 10^{28} \mathrm{erg}$ for the burst's lifetime of 36 minutes. This compares with the value $5 \times 10^{28} \mathrm{erg}$ derived with a different method for the IB studied by Peter et al. (2014). We also note that a typical energy for a X-class flare is $10^{32} \mathrm{erg}$ (e.g., Emslie et al. 2005).

\subsection{Magnetic Environments and Signatures}

The UV bursts reported in the literature are generally characterized by rapid evolution of small-scale magnetic elements on the photospheric surface, often evidenced in the $45 \mathrm{sec}-$ ond cadence LOS magnetograms from HMI but sometimes requiring better resolution and sensitivity. Three types of magnetic environment are recognized to harbor UV bursts:

1. Emerging flux regions (EFRs) in complex active regions. Small flux elements in these display fast streaming motions; cancellation occurs regularly.

2. Moving magnetic features (MMF) in sunspot moats. These can have opposite polarity to the spot in sea-serpent patterns (Harvey and Harvey 1973) with cancellation against same-polarity features.

3. Light bridges (LB). Elongated features with highly-sheared magnetic field that cross sunspot umbrae, or occur close to them (Solanki 2003).

EFR examples include the events in Peter et al. (2014), Vissers et al. (2015), Toriumi et al. (2017), Zhao et al. (2017) and Rouppe van der Voort et al. (2017). Gupta and Tripathi (2015) and Chitta et al. (2017) studied MMF events. Bursts associated with an LB were discussed by Toriumi et al. (2015b) and Tian et al. (2018a).

HMI magnetograms are shown in the rightmost column of Fig. 2. Events (a)-(c) are located within $1^{\prime \prime}$ of small negative polarity features with vertical flux densities of around 200-400 G. Event (d) from Toriumi et al. (2015b) is located to the north side of a light bridge running east-west and does not show an obvious compact magnetic feature, but the authors noted a continuous supply of field with LOS flux densities of 200-400 G within the light bridge that kept driving brightenings. Hinode Spectropolarimeter data showed that this field was highly inclined with horizontal flux densities of around $1000 \mathrm{G}$. This highlights the importance to obtain the full vector magnetic field when studying UV burst evolution.

Light bridges are believed to be convective intrusions into strong background magnetic field and have mostly horizontal field (Leka 1997). Reconnection between this field and the surrounding vertical field generates EB-like phenomena and surges of cool plasma (Roy 1973). Recent, high-resolution $\mathrm{H} \alpha$ data from the SST show that the surges resolve into a "fan" of many jets side-by-side (Robustini et al. 2016). IRIS data have demonstrated that UV bursts are rooted in LBs at the bases of the surges (Toriumi et al. 2015a; Hou et al. 2017; Tian et al. 2018a). Since reconnection events are constantly driven by the convection in the LB, repeated bursts and surges can be sustained for hours but individual events are short-lived (Asai et al. 2001).

The line-of-sight (LOS) magnetograms from HMI are valuable for studying the magnetic field evolution of UV bursts, but the precise magnetic field topology requires vector magnetograms and/or extrapolation of the field into the corona. Vector magnetograms are available from HMI at 720 second cadence, but the noise level for the transverse field is about $100 \mathrm{G}$ compared to about $10 \mathrm{G}$ for the LOS magnetograms, not sufficient for the small field concentrations that typically underly or cause UV bursts. 
Fig. 6 A cartoon from Georgoulis et al. (2002) indicating how an Ellerman bomb can be triggered by converging horizontal flows in the photosphere. Reproduced by permission of the AAS
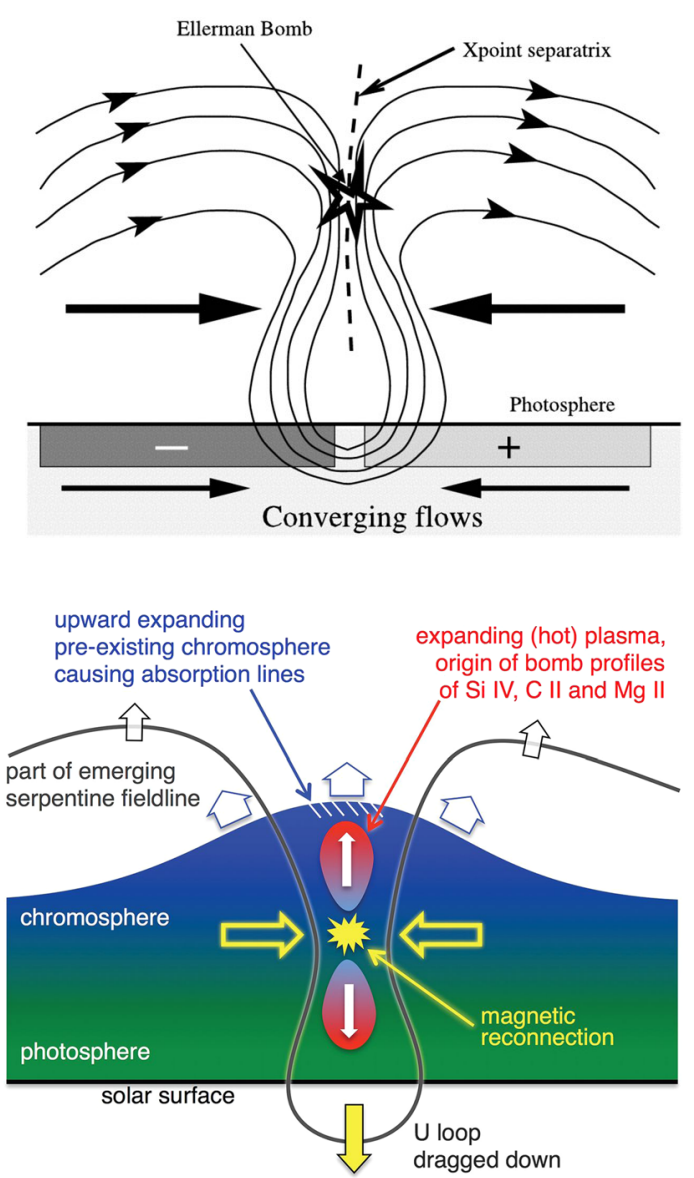

Fig. 7 A cartoon for the formation of an IRIS bomb. This scenario follows that of Fig. 6 , but has additional features to account for the IRIS signatures, as indicated on the figure. From Peter et al. (2014). Reprinted with permission from AAAS

A particular scenario believed important for EBs and UV bursts is U-loop reconnection, in which reconnection takes place in the arms of a U-shaped magnetic field line (Figs. 6 and 7). Such a field configuration can occur where flux emerges through the photosphere and mass-loading at the center of the loop prevents it from rising into the atmosphere. When viewed in a LOS magnetogram, the field shows two distant footpoints and between them an apparent, close bipole where the field is U-shaped rather than having the $\Omega$ shape of normal field configurations. Such configurations are sometimes referred to as "bald patches"- see the magnetic field lines at the photosphere in Fig. 6. The difference with $\Omega$ sea-serpent configurations can only be recognised from vector magnetograms of sufficient quality to establish that the field connects the two polarities underneath rather than above. This was done by Georgoulis et al. (2002) for an emerging flux region observed in the Flare Genesis balloon flight, leading them to suggest the U-shaped loop reconnection scenario for EBs (Fig. 6) that was later adopted also by Peter et al. (2014) for IBs (Fig. 7) Due to this nature of the field configuration the reconnection necessarily occurs in the low atmosphere.

A different scenario was presented by Chitta et al. (2017), who were able to identify a fanspine magnetic topology for a MMF UV burst by applying a magnetofrictional relaxation technique to HMI LOS magnetograms. This technique begins with a potential field extrapolation from an initial magnetogram, which is then evolved to a series of non-linear force-free 
field states by inputting the subsequent magnetograms as new boundary conditions. The null point of the fan-spine system was found to be at $500 \mathrm{~km}$, and the reconnection driving the UV burst was suggested to be due to shearing at the null point, driven by the motion of the MMF.

Zhao et al. (2017) and Tian et al. (2018b) combined vector magnetogram data from HMI with a MHD relaxation technique (Zhu et al. 2013, 2016) to investigate the magnetic environment of UV bursts. Zhao et al. (2017) distinguished UV bursts associated with bald patches and those with flux cancellation. Tian et al. (2018b) also found that some UV bursts were associated with bald patches for a different emerging flux region, but most of the bursts were not. A better correspondence was found with locations with a high squashing factor that are often associated with currents and magnetic reconnection with regard to the generation of flares.

The evidence from the magnetic field extrapolations is that reconnection takes place at heights of 0.5-1.0 Mm (Chitta et al. 2017; Tian et al. 2018b). Further evidence for these low heights comes from the weakness or absence of coronal emission (Sect. 4.8) and the appearance of the narrow cool-gas blends on the IB profiles. Note that these blends do not require the presence of cool gas on top of (and part of) the bomb as suggested by Peter et al. (2014), but may originate in cool gas along a slanted LOS to the bomb base as in Fig. 2 of Rutten (2016). Such gas then also absorbs any coronal EUV radiation from the burst at wavelengths below the hydrogen photoionization edge at $912 \AA$.

High-quality vector magnetic field measurements are highly desirable for UV bursts, both high spatial resolution and high sensitivity. Although current ground-based instruments are capable of much better measurements than HMI, the polarization data are much more susceptible to bad seeing so that homogeneous good-quality sequences covering the entire duration of UV bursts have not yet been obtained. This is one area where the 4 meter Daniel K. Inouye Solar Telescope (DKIST) should offer large improvement.

Unipolar magnetic field regions show a different type of intensity enhancement, with examples presented by Toriumi et al. (2017) for a small EFR. In addition to the bursts in the center of the EFR, which were interpreted as bald patch events, a set of brightenings above the unipolar patches at the edge of the EFR were identified. These were interpreted as shockheating events driven by plasma flowing down the legs of the rising arch filament system. The unipolar region events showed weaker Si IV intensities than the bald patch bursts, with an enhancement of only around five compared to quiet regions; these may not generally qualify as UV bursts.

\subsection{Connection to Ellerman Bombs}

Peter et al. (2014) recognized the connection between IBs and EBs by naming the events "bombs". Their U-loop reconnection scenario was partly based on the similar one for EBs by Georgoulis et al. (2002). However, without access to simultaneous $\mathrm{H} \alpha$ observations, the authors could not make the IB-EB connection. (The later work of Grubecka et al. 2016, on the same data-set could, however, make a connection to EBs for IBs 3 and 4 based on signatures in the Mg II lines-see below.)

Direct IB-EB connections were demonstrated by Vissers et al. (2015), Kim et al. (2015) and Tian et al. (2016), who each had access to simultaneous ground-based $\mathrm{H} \alpha$ data and found examples of EBs that had a clear IB signature, i.e., intense, strongly-broadened Si IV emission lines with superimposed narrow cool-gas blends. Tian et al. (2016) identified 10 IBs based on the presence of broadened Si IV lines with such superimposed absorption features. Three IBs were clearly matched with EBs, four had no EB signature, the remaining 
three were ambiguous. They also noted that while the IRIS slit crossed about 30 EBs only 6 had an IB signature.

Libbrecht et al. (2017) used $\mathrm{H} \beta$ to identify $21 \mathrm{EBs}$, and found four of the events showed wing enhancements in the $\mathrm{He} \mathrm{I} \mathrm{D}_{3}$ and $\lambda 10830$ lines-the first reported EB signatures in these lines-suggesting temperatures in excess of $20 \mathrm{kK}$. Two of the four EBs were observed through the IRIS slit and enhanced Si IV emission was found for each, with one showing IB-like line profiles. This suggests that EBs displaying hot emission may also have signatures in the He I lines, which could be significant for future ground-based observations (e.g., DKIST).

As simultaneous, high-resolution, spectroscopic $\mathrm{H} \alpha$ data are rarely available for IRIS observations some authors have sought to find EB identifiers in the IRIS data themselves. Grubecka et al. (2016) found that 1D radiative transfer models that fit the $\mathrm{H} \alpha$ wings of EBs may also fit the wings of the $\mathrm{Mg}$ II lines, and therefore used $\mathrm{Mg}$ II $\mathrm{h}$ line wing emission to find EBs in their IRIS data-set. They found that samples at -3.5 and $+1.0 \AA$ ( -374 and $+107 \mathrm{~km} \mathrm{~s}^{-1}$ ) are suited for identifying EBs, and subsequently identified 74 events that were a factor two brighter than their surroundings. Around $10 \%$ of these events had a signature in the SJI $1400 \AA$ A channel.

Tian et al. (2016) used narrow-band filtergrams in the wings of $\mathrm{H} \alpha$ to identify EBs and found that averaging images obtained at $\pm 1.33 \AA\left( \pm 143 \mathrm{~km} \mathrm{~s}^{-1}\right)$ from the line core of $\mathrm{Mg}$ II $\mathrm{k}$ is a good proxy for $\mathrm{H} \alpha$ EBs. Another method for detecting EBs with Mg II was suggested by Hong et al. (2017b) who integrated a region $\pm 0.25 \AA$ around the two members of the Mg II triplet lines at 2798.75 and $2798.82 \AA$, and found a good correlation with $\mathrm{H} \alpha$ wing enhancements found with the Fast Imaging Solar Spectrograph on the Goode Solar Telescope.

The ubiquitous availability of AIA full-disk images offers another avenue for finding EB signatures, with the AIA 1700 and $1600 \AA$ A passbands the most promising. Rutten et al. (2013), Vissers et al. (2013) and Vissers et al. (2015) compared $\mathrm{H} \alpha$ observations from SST/CRISP with AIA and found good correlations between $\mathrm{H} \alpha$ EBs and AIA mid-UV brightenings. The last paper set as EB criterion $\geq 8 \sigma$ above the mean $1700 \AA$ intensity over the whole active region. Further study (G. Vissers 2018, private communication) suggests that $1700 \AA$ is the best of the AIA passbands for identifying EBs, allowing recovery of nearly $20 \%$ of the $\mathrm{H} \alpha$ EBs when using a $\geq 5 \sigma$ above mean intensity threshold, combined with a lower lifetime threshold of 1 minute and size limits of 1-16 pixels. Optimizing instead for the number of AIA candidates that are indeed $\mathrm{H} \alpha$ EBs (reaching over $60 \%$, though only recovering about $5 \%$ of the total $\mathrm{H} \alpha \mathrm{EB}$ population) requires a higher brightness threshold $(\geq 9 \sigma)$ and stricter size constraints (1-9 pixels), but keeping the same lifetime threshold. While complete one-to-one correspondence is thus not possible, the $1700 \AA$ brightenings are of interest in their own right as already noted by Rutten et al. (2013).

Finally we emphasize that the results of Grubecka et al. (2016) and Tian et al. (2016) suggest that $10-20 \%$ of EBs have an IB signature. Tian et al. (2016) have also suggested that $30-60 \%$ of UV bursts are co-spatial with EBs. Since Sect. 5 below shows that it is hard to achieve both EB signatures and UV burst signatures from a single reconnection event when modeling EBs and IBs, then obtaining improved statistics on simultaneous EBs and bursts is desirable.

\subsection{Mini Flare Ribbons}

Our definition of UV bursts in Sect. 2 excludes bright kernels in flare ribbons, but active regions can also display what we call "mini flare ribbons" that are small in size (say, 10" 

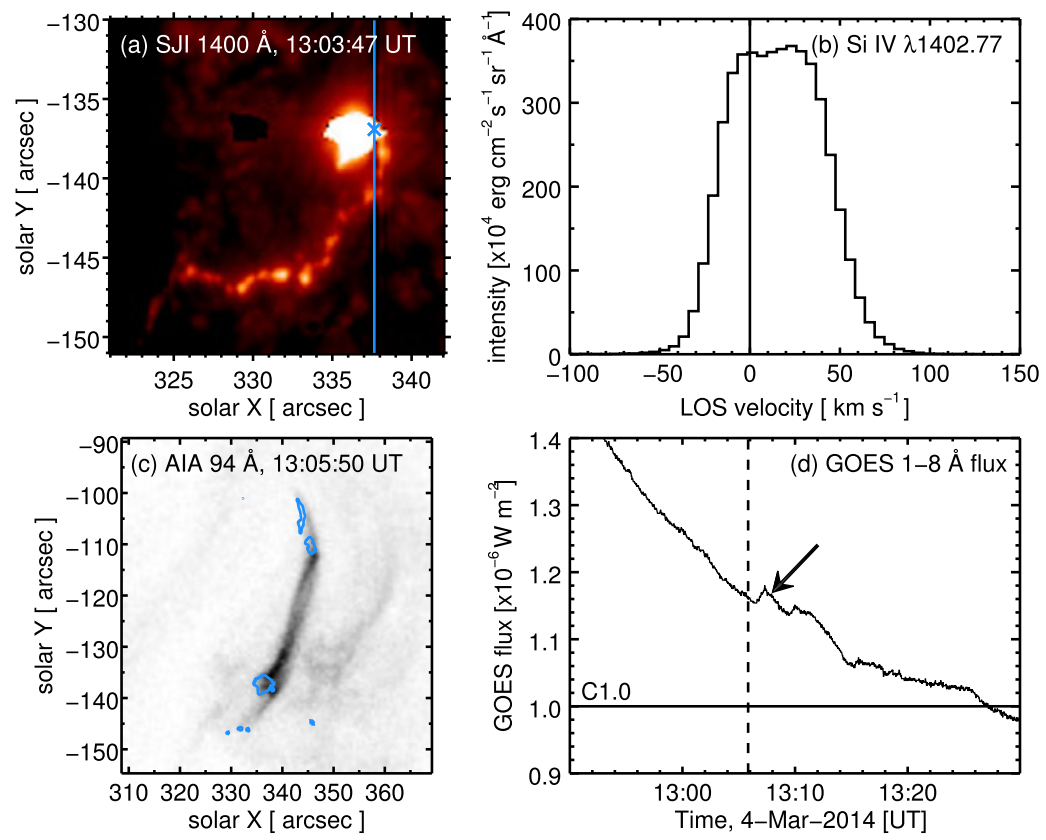

Fig. 8 An example of a mini flare ribbon from 2014 March 4. Panel (a) shows an IRIS SJI $1400 \AA$ image with a logarithmic intensity scale. The blue vertical line shows the position of the IRIS slit, and the cross the location corresponding to the spectrum shown in panel (b), where Si IV $\lambda 1402.77$ is shown. Panel (c) shows an AIA $94 \AA$ image (linear intensity scale) from two minutes later, with contours showing the SJI $1400 \AA$ intensity from panel (a) at a level of $1000 \mathrm{DN} \mathrm{s}^{-1}$. Note the displayed region is larger than for panel (a). Panel (d) shows the GOES 1-8 A flux with the time of the AIA image indicated with the vertical dashed line. An arrow indicates a feature that may correspond to the mini flare (see main text)

or less) and have either no or very weak signal in the 1-8 $\AA$ channel of the Geostationary Operational Environmental Satellite (GOES) X-ray monitor. The Si IV intensities of such events are comparable to UV bursts, but a key distinguishing feature is that the lines are mostly red-shifted, consistent with the profiles seen in most normal flare ribbons. The ribbons probably correspond to coronal nano- or micro-flares that would be visible at X-ray or EUV wavelengths.

The only example in the IRIS literature is the event described by Bai et al. (2016) which took place in the penumbra of a small sunspot. Multiple transient brightenings took place along a ribbon of length $10^{\prime \prime}$, with the brightest comparable with the Peter et al. (2014) IBs. The Si IV profiles were mostly Gaussian shaped and red-shifted by $15-20 \mathrm{~km} \mathrm{~s}^{-1}$. The ribbon also exhibited coronal emission in the AIA channels, which is not typical of UV bursts (Sect. 4.8).

Similar events have been found by the ISSI team and an example is shown in Fig. 8. The IRIS slit crossed a very intense, compact brightening that is visible in panel (a) and could be interpreted as a UV burst. Note the "chain" of weaker brightenings extending from the main brightening that is reminiscent of a flare ribbon. The Si IV $\lambda 1402.77$ line profile is predominantly red-shifted and significantly narrower than the profiles of Fig. 2. The line's amplitude, however, is comparable to those of Figs. 2 and 5. About two minutes after the IRIS image was taken, the AIA $94 \AA$ bandpass (panel c) shows a loop running northwards from the location of the intense IRIS brightening to another strong brightening (not shown 

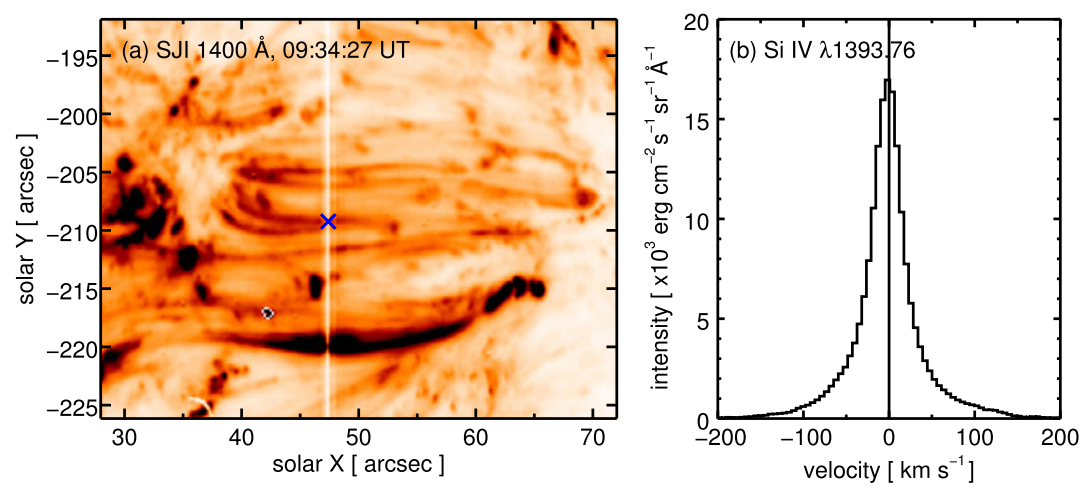

Fig. 9 Example Si IV profile from a dynamic TR loop. The left panel shows an IRIS SJI $1400 \AA$ image of AR 11916 from 2013 December 6. A linear, inverted intensity scale is used. A blue cross denotes the spatial location from which the spectrum in the right panel was taken

in panel a). This loop is clearly filled with plasma at $\sim 10 \mathrm{MK}$ due to chromospheric evaporation, as expected from the standard flare model. Panel (d) shows the GOES X-ray flux at this time, where a small peak is seen shortly after the time of the AIA image. It is not clear if this peak arises from the event as the GOES flux is an average over the entire solar disk, but if it is then the energy of an A-class flare is implied. This event has been studied in more detail in a recent paper of Gupta et al. (2018).

To distinguish a UV burst from a mini flare ribbon, the key features to check are (i) an extended ribbon structure, (ii) a predominantly red-shifted line profile, and (iii) loop emission in the "hot" AIA channels at 94 and $131 \AA$ that would imply chromospheric evaporation has taken place.

Bai et al. (2016) referred to their event as a nanoflare ribbon after estimating the thermal energy from AIA imaging. We prefer the term "mini flare ribbon" in order to cover a wider range of energies, and suggest that there is a need for a survey of such events to investigate how they compare with larger flares.

\subsection{Dynamic Loops (Arch Filament Systems)}

Another class of events in SJI $1400 \AA$ images that show strong UV-line emission but not considered by us to be UV bursts are dynamic TR loops. These are mostly seen in emerging flux regions, and seem to be analogous to the fibrils of arch filament systems that are seen in absorption in $\mathrm{H} \alpha$ (Bruzek 1967). Examples have been presented by Yan et al. (2015), Huang et al. (2015) and Huang et al. (2017). The loops span the emerging flux region and are typically 5-40" long. Propagating intensity fronts in SJI $1400 \AA$ image sequences suggest flows along these loops are common. The line profiles often show a relatively narrow central component with weaker, but very extended wings. We show an example from the data-set studied by Yan et al. (2015) in Fig. 9. These authors showed in their Fig. 3 a more intense loop spectrum with a complex line profile. We consider Fig. 9 to be a more representative dynamic loop spectrum, and Fig. 5 from Huang et al. (2017) shows a similar example from a different data-set. Post-flare loop arcades can also exhibit similar profiles, such as those shown in Fig. 4 of Brannon (2016). The Si IV intensities of dynamic loops are generally lower than those of the most intense UV bursts. For example the peak amplitude of Si IV $\lambda 1402.77$ for the event shown in Fig. 9 is $9000 \mathrm{erg} \mathrm{cm}^{-2} \mathrm{~s}^{-1} \mathrm{sr}^{-1} \AA^{-1}$, which can 
be compared with the numbers shown in blue in Figs. 2 and 5. Zhao et al. (2017) applied magnetic field extrapolation to an emerging flux region (see also Sect. 4.3), attributed the low-lying field lines in their extrapolation to an arcade of dynamic loops seen in SJI $1400 \AA$, and associated the latter with reconnection between the emerging flux and the overlying magnetic field at quasi-separatrix layers (QSLs). Previously, Georgoulis et al. (2002) had identified the connection between QSLs and EBs for an emerging flux region, and they also identified bright fibrils in the TRACE $1600 \AA$ channel that they interpreted as due to bright $\mathrm{C}$ IV emission. These were likely the equivalent of the Si IV dynamic loops.

\subsection{Flaring Fibrils}

A study of EBs identified from the Flare Genesis Experiment revealed co-spatial brightenings and dynamic loops in the TRACE $1600 \AA$ channel (Georgoulis et al. 2002; Schmieder et al. 2004). The loops were referred to as "flaring arch filaments", and Vissers et al. (2015) used this notation to describe compact brightenings in AIA $1600 \AA$ images, similar to EBs but with obvious elongated morphology and proper motion along filamentary strands that they interpreted as due to C IV line emission lines in this passband. Rutten (2016) subsequently proposed that "flaring active-region fibrils" (FAFs) is a better name as it avoids confusion with the dynamic loops of arch filament systems referred to in the previous section.

Vissers et al. (2015) compared AIA FAFs with IRIS data and found intense, IB-like Si IV profiles at the locations of the $1600 \AA$ brightenings, with jets or loops in the SJI $1400 \AA$ images and shell-like fronts expanding away from them in the hotter AIA passbands. To highlight the difference between the dynamic loops described in the previous section with FAFs, we show a SJI $1400 \AA$ image from the 2014 June 15 dataset studied by Vissers et al. (2015) in Fig. 10a. There are many dynamic loops in this image, and they can be seen to have a generally smooth variation in their intensities from footpoint to footpoint. The FAFs are the brightest loops in the image which appear to be connected to compact brightenings. Note that the brightenings can be identified as discrete features - the inset image of Fig. 10(a) shows one of the FAF brightenings on a linear intensity scale, revealing a distinct brightening. In particular, the brightenings are not simply small segments that brighten during the loop's evolution. Si IV $\lambda 1402.77$ spectra from three locations are shown in panels (c)-(e). (c) and (e) correspond to FAF brightenings, and show the broad, very intense profiles of IBs (compare with Fig. 2), although (e) does not show the cool absorption blends. Panel (d) shows a profile from a fibril that connects to the FAF brightening, and it is more comparable to the dynamic loop profile shown in Fig. 9, although the intensity is an order of magnitude larger. The FAF brightenings we consider to be UV bursts, and a plausible interpretation is a low-lying reconnection event that is able to connect to the overlying arch filament system and deposit heated plasma in certain loops.

Another event in the literature that we consider to be a FAF is the one shown in Fig. 13 of Huang et al. (2015), which was described as the footpoint of two interacting loop systems that exhibited explosive event line profiles.

To summarize the terminology, the FAF is the combination of the loop (fibril) and the burst that evolve together.

\subsection{Coronal Signatures}

Peter et al. (2014) noted that the four IBs they observed did not have a signature in the coronal AIA passbands. This seems to be a common feature of UV bursts, but not an absolute 


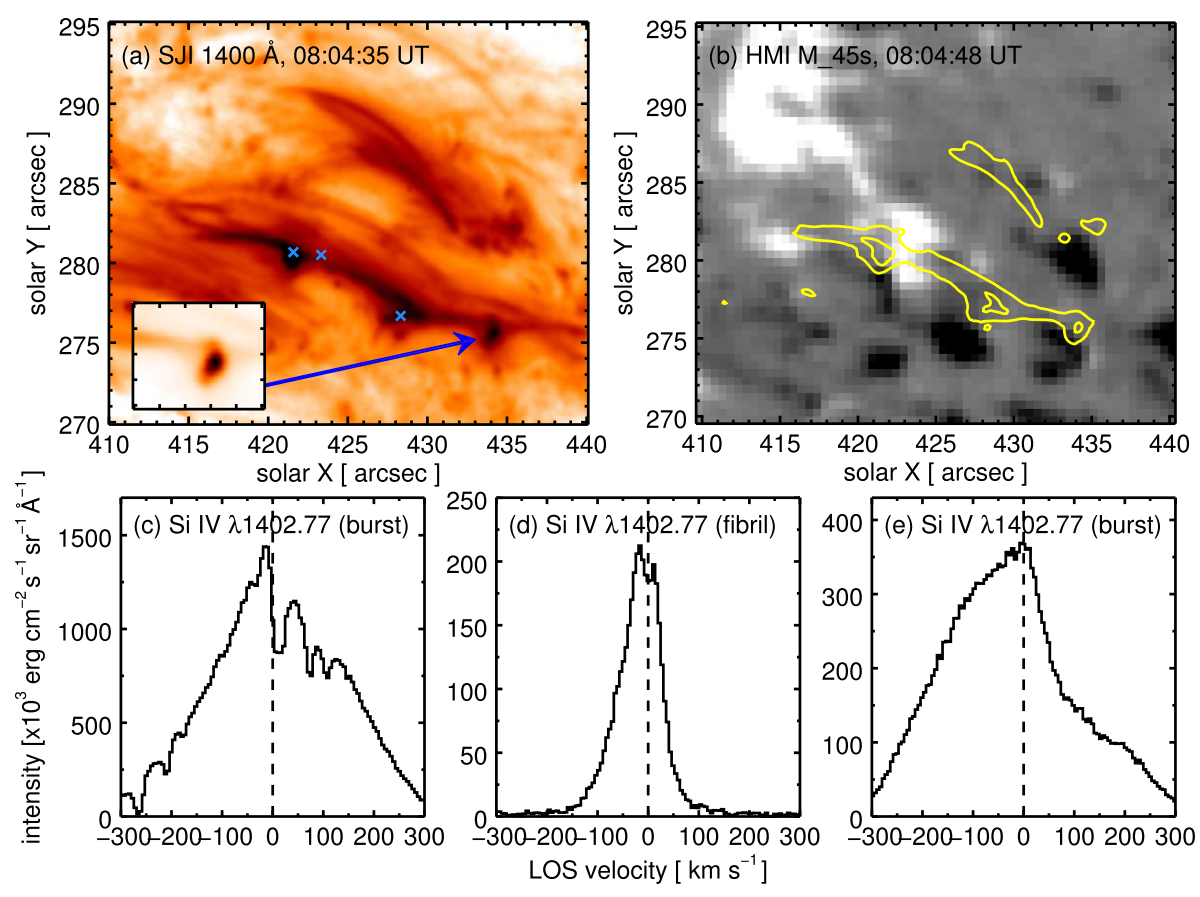

Fig. 10 Example of a flaring arch fibril. Panel (a) shows an IRIS SJI $1400 \AA$ image of AR 12089 from 2014 June 15. A logarithmic, inverted intensity scale is used, and the inset shows a section of the image containing a FAF with a linear intensity scale. Panel (b) shows a co-temporal LOS magnetogram from HMI, scaled between -500 and $+500 \mathrm{G}$. Yellow contours are derived from the SJI image, with levels of 300 and $2000 \mathrm{DN} \mathrm{s}^{-1}$. Panels (c), (d) and (e) show three Si IV $\lambda 1402.77$ spectra corresponding to the left, middle and right locations indicated by the blue crosses in panel (a)

rule. For example, the event of Gupta and Tripathi (2015) showed weak AIA signals, allowing the authors to derive a differential emission measure curve, and Vissers et al. (2015) described thin long arcs seen in the hotter AIA diagnostics spreading away from FAF sites.

The weakness or absence of co-spatial coronal emission either implies that the bursts are not heated beyond about $10^{5} \mathrm{~K}$, or that the EUV emission is blocked by overlying cool plasma with sufficient neutral hydrogen and helium that all lines below $912 \AA$ are strongly attenuated. IRIS does observe a coronal line above $912 \AA-F e$ XII $\lambda 1349.40$, with $T_{\max }=1.6 \mathrm{MK}$ - but this line is very weak and has not yet been reported from a UV burst observation.

\section{UV Burst Modeling}

Our interpretation of UV bursts as magnetic reconnection events in the low atmosphere means there are three components to modeling that must be considered: (1) the atmospheric evolution that leads to reconnection at low heights in the atmosphere; (2) the physics associated with the reconnection current sheets; and (3) the effect of dynamics and heating on spectral line profiles. Our definition of a UV burst requires the simulations to produce a compact, very intense, flickering brightening in a TR line (with $T_{\max } \sim 100 \mathrm{kK}$ ). Since most 
UV bursts also show the complex line profiles of IBs and many also the cool-line absorption blends, then these are additional tests for the models to pass.

UV bursts are relatively new discoveries so simulations directly focused on them are few, but a number of works have considered how simulations relevant to EBs may produce UV bursts. In particular, over the past 20 years increasingly sophisticated codes for modeling flux emergence have been performed and demonstrated to produce the U-loop reconnection that has been suggested for EBs and IBs (Figs. 6, 7). The physics of magnetic reconnection current sheets has also been studied extensively, and may be particularly relevant to the often complex line profiles of UV bursts. Another focus of modeling has been on whether $1 \mathrm{D}$ radiative transfer models that are able to reproduce the profiles of strong chromospheric lines from EBs are consistent with UV bursts.

1D modeling of EBs has a history going back to Kitai (1983), with the most basic aim to reproduce the wings-only brightening of $\mathrm{H} \alpha$. The method involves the perturbation of a plane-parallel atmosphere, usually by the insertion of a hot component near the temperature minimum region (heights of $\approx 450 \mathrm{~km}$ ). NLTE radiative transfer is included and profiles of strong lines such as $\mathrm{H} \alpha$, Ca II $\mathrm{H} \& \mathrm{~K}$ and $\mathrm{Mg}$ II h \& $\mathrm{k}$ are modeled. Recent work in this vein includes Berlicki et al. (2010), Berlicki and Heinzel (2014), Grubecka et al. (2016) and Fang et al. (2017), and the more sophisticated 2D NLTE modeling by Bello González et al. (2013). Cloud modeling introduces an additional plasma component to simulate the effect of absorption by overlying chromospheric fibrils on the line core, and a recent example is the work of Hong et al. (2017b). Comparisons of the models with observations typically constrain the hot component to be about $100-3000 \mathrm{~K}$ above the background temperature (e.g., Li et al. 2015; Grubecka et al. 2016). Note that, since the models are focused on effects at the temperature minimum region, the atmospheres are usually truncated at $20000 \mathrm{~K}$ or lower, well below the $T_{\max }$ of Si IV.

The discovery of IBs led Fang et al. (2017), Reid et al. (2017) and Hong et al. (2017a) to investigate whether the EB models could be modified to produce Si IV emission. Fang et al. (2017) considered temperatures of the hot component up to $15 \mathrm{kK}$, but then the chromospheric signatures were not consistent with the EB observations. Both Reid et al. (2017) and Hong et al. (2017a) used the RADYN code (Carlsson and Stein 1997), which couples radiative transfer with hydrodynamics, to allow the plasma to respond to the heating. Again the authors found that it was not possible to reconcile Si IV emission with the chromospheric line profiles of EBs. We highlight that, as discussed in Sect. 4.4, only 10-20\% of EBs have been found to have a hot UV burst signature, thus the failure of the 1D models to account for the hot emission does not invalidate the models for the much more common, cooler events. In addition, it is possible that non-equilibrium effects such as $\kappa$ electron distributions can cause Si IV to be formed at much lower temperatures (Dudík et al. 2014). A similar effect may also arise if EBs are formed at sufficiently high density that Si IV has near-Saha-Boltzmann opacity, giving a formation temperature of 10-20 kK (Rutten 2016). However, within this cooler regime no attempt has been yet made to reproduce the nonvisibility of EBs in the $\mathrm{Na}$ I D and Mg I b lines, already mentioned by Ellerman (1917) and confirmed by Rutten et al. (2015), which may be difficult to reconcile with 1D temperature humps covering the formation heights of these lines.

There are a number of simulations that focus solely on the physics of magnetic reconnection current sheets in the solar atmosphere. They do not investigate the wider plasma evolution that leads to the sheet formation, but they do resolve the current sheet at higher resolution than large-scale MHD codes. Ni et al. $(2015,2016)$ investigated plasma heating in current sheets located at atmospheric heights of 100 to $1000 \mathrm{~km}$, and with horizontal and vertical orientations. Plasmoids are formed in the current sheet, and heating occurs where 


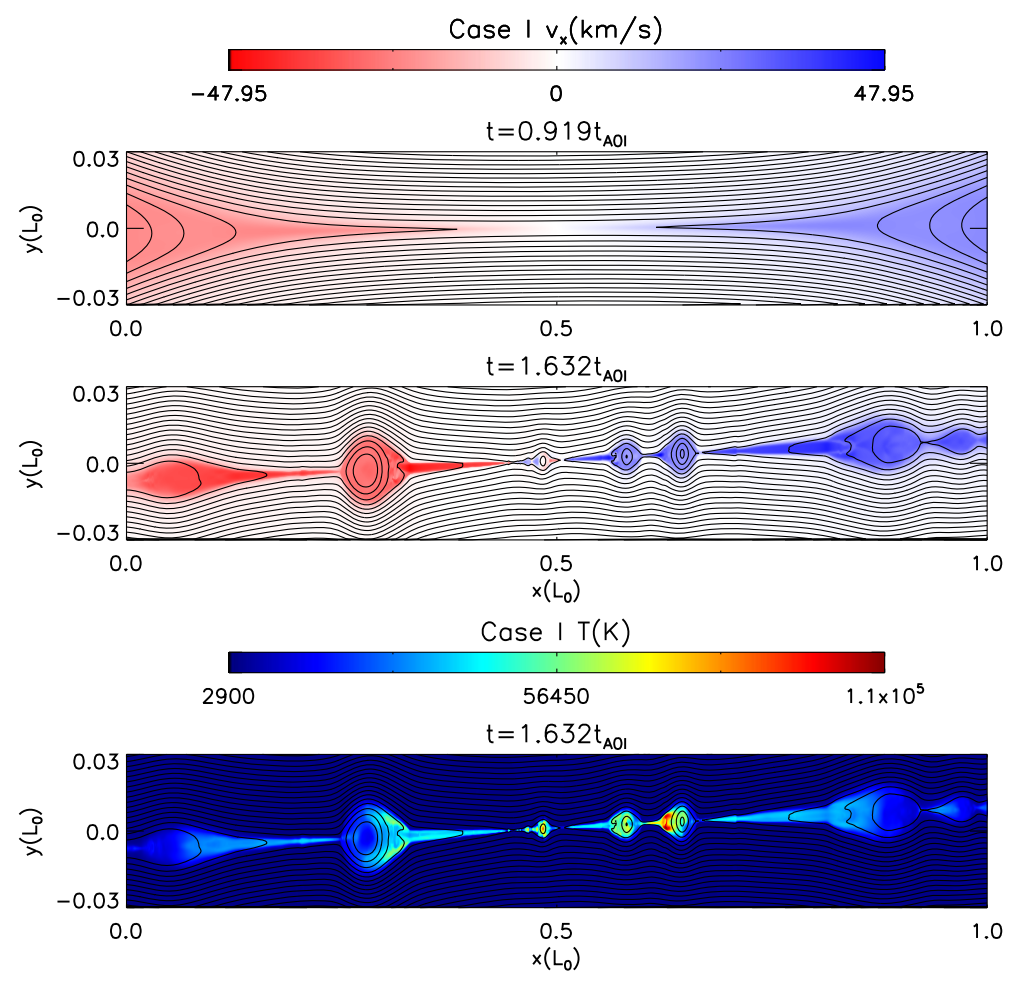

Fig. 11 Images from the current sheet simulation of $\mathrm{Ni}$ et al. (2016). The upper panel shows the configuration prior to plasmoid formation, with velocity in the $X$-direction ( $v_{x}$, parallel to the current sheet) plotted; contours show the magnetic field. The middle panel shows $v_{x}$ at a later time after plasmoids have formed, and the lower panel shows temperature at this time, revealing $100 \mathrm{kK}$ temperatures in the plasmoids. Reproduced by permission of the AAS

slow-mode shocks from the reconnection site interact with the plasmoids (Fig. 11). They found that the plasma $\beta$ (ratio of plasma pressure to magnetic pressure) is crucial in determining if UV burst temperatures are produced: $\beta \lesssim 0.1$ enables heating to $80 \mathrm{kK}$ (the temperature of formation of Si IV). The further work of $\mathrm{Ni}$ et al. (2018) found that the inclusion of non-equilibrium ionization in current sheet simulations results in a faster reconnection rate but smaller temperature increases, potentially affecting the formation of Si IV.

Innes et al. (2015) also studied current sheet dynamics in relation to burst-like events recorded from IRIS. They highlighted that the break-up of a current sheet into plasmoids separated by magnetic field x-points at which particles are accelerated can give rise to complex velocity distributions. Modeling the Si IV emission lines integrated along the current sheet was found to give realistic, IB-like profiles. They also highlighted that a relatively small change to the line-of-sight had a significant effect on the profiles.

Another 2.5D simulation that produced current sheet plasmoids, but this time as part of the wider evolution of solar atmospheric structures was presented by Nóbrega-Siverio et al. (2017) and Rouppe van der Voort et al. (2017). The interaction of an emerged bipole with pre-existing magnetic field was simulated, leading to a surge and a current sheet. The latter occurred where the "dome" of the emerged flux pushed against opposite polarity field, and the simulation resolution was sufficient to resolve plasmoids. Figure 12 shows images taken from these papers. The authors chose the line-of-sight indicated by the diagonal lines in the 

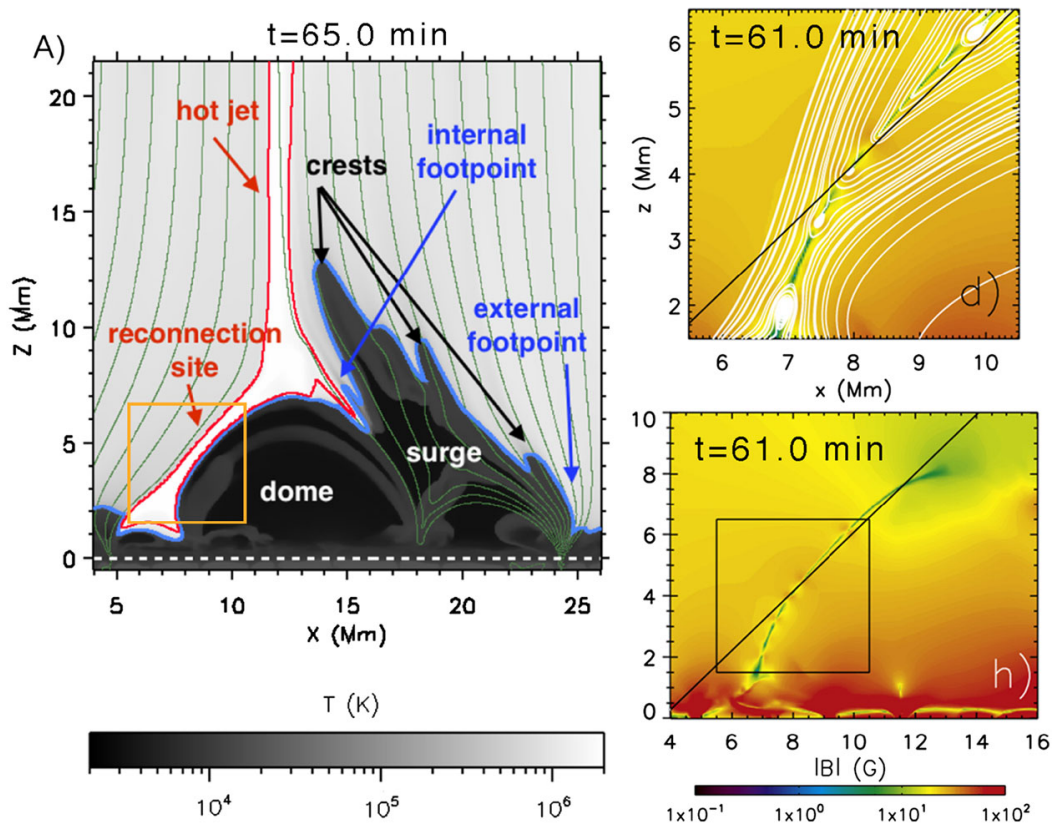

Fig. 12 Still frames from a 2.5D simulation of emerging flux. The left panel (from Nóbrega-Siverio et al. 2017) shows a temperature map with green contours representing magnetic field, the blue contour shows the layer at which Si IV is formed $(80 \mathrm{kK})$, and the red contour shows plasma at $1.2 \mathrm{MK}$. Key features of the model are labeled. The orange box highlights the location of the current sheet, which is shown in progressively greater detail in the bottom-right and top-right panels, which both show the magnetic field strength. Magnetic field contours on the top-right panel emphasize the location of the plasmoids along the current sheet. These panels are from Rouppe van der Voort et al. (2017) at a slightly earlier time than the left panel. Reproduced by permission of the AAS

two right panels, and they found that complex, IB-like Si IV profiles were produced from the multiple plasmoids along the current sheet. Note that the reconnection in this scenario is not the U-loop reconnection discussed in Sect. 4.3, but that more commonly associated with jets (e.g., Fig. 8 of Shimojo and Shibata 2000). This is also the scenario inferred by Chitta et al. (2017) for a MMF burst.

There are many time-dependent simulations of emerging flux in the literature (see review of Schmieder et al. 2015), and a particular issue relevant to UV bursts and EBs is the occurrence of U-loop reconnection (Sect. 4.3 and Figs. 6, 7). This has been demonstrated to arise naturally on account of the Parker buoyancy instability acting on the flux and giving rise to so-called serpentine field lines. A 2D resistive MHD simulation of an emerging flux sheet was presented by Isobe et al. (2007), and it showed plasma heating and flows at the sites of U-loop reconnection that were identified with EBs. A similar result was found by Archontis and Hood (2009) but for a fully-3D resistive MHD simulation of flux sheet emergence. Further advances were made by Tortosa-Andreu and Moreno-Insertis (2009) and Cheung et al. (2010), who performed 3D simulations for the emergence of twisted cylindrical and semi-torus flux tubes, respectively, demonstrating U-loop reconnection in both cases. The latter work effectively demonstrated that EBs can occur during the formation of a bipolar active region.

These latter two simulations both used the MURaM code (Vögler et al. 2005) and included simplified radiative transfer effects. More recently the code has been applied to the 
case of an emerging flux sheet by Danilovic (2017), but with a more sophisticated treatment of radiative transfer, including detailed $\mathrm{H} \alpha$ synthesis. The model produces realistic EB signatures, but the atmosphere does not extend beyond the chromosphere and so UV bursts are not produced.

The Bifrost code of Gudiksen et al. (2011) does extend to the chromosphere and corona, allowing the evolution of flux emergence to be studied in these layers. This was done in full 3D by Hansteen et al. (2017) who explicitly address EBs, UV bursts including IBs, and microflares. It includes spectral synthesis of $\mathrm{H} \alpha, \mathrm{Mg}$ II h \& k, and the Si IV $1393.7 \AA$ line including the Ni II blend for selected simulation snapshots. There are many striking agreements between the simulated and observed appearances of both EBs and UV bursts. In the simulation the EBs are photospheric in origin and remain generally under $10000 \mathrm{~K}$; the IB-like UV bursts are chromospheric and reach higher temperatures although their total energy-release content is far less. The microflares go higher up and reach coronal temperatures. In this simulation they are all the result of reconnection between the legs of draggeddown U-loops and expanding $\Omega$-loops that are part of serpentine flux emergence. Unlike the $2 \mathrm{D}$ codes discussed earlier, the simulation resolution is not sufficient to resolve plasmoids in the current sheets, but broad Si IV line profiles are produced from bi-directional flows at the reconnection sites. A remaining discrepancy is that the simulated EBs show Si IV emission only barely or none at all, whereas this has been observed for 10-20\% of EBs. This version of the Bifrost code did not yet account for ion-neutral separation (ambipolar diffusion) whereas tests have shown in the meantime that this can substantially increase the rate of reconnection (Martínez-Sykora et al. 2017), which may impact the predicted emissions of EBs.

\section{Summary}

The IRIS instrument has revealed a set of intense, transient, compact brightenings that occur in active regions and are visible in the resonance lines of Si IV. In this work we advocate that these events be referred to as UV bursts. The definition of UV bursts is given in Sect. 2 and it includes the set of events that have been called IRIS bombs in the literature. The latter were introduced by Peter et al. (2014), and are defined through their spectroscopic signature in the Si IV lines.

Studies of UV bursts are ongoing, but they are widely believed to be small-scale reconnection events occurring at heights from the photosphere to the upper chromosphere. As the events reach TR temperatures then they have special advantages for investigating reconnection in the solar atmosphere. The TR is thin (typically 10's of kilometers) and so the large energy output from reconnection gives a very strong signal in TR lines that overwhelms the background emission and allows fine details in the line profiles to be studied. In contrast a reconnection event in the corona takes place within a volume that is 10 's of megameters thick, and thus gives a relatively subdued signal unless it is a significant size flare. The TR has a number of very strong lines in the far ultraviolet where instrument sensitivity, spectral and spatial resolution are generally higher than for the EUV and X-ray regions, where most coronal lines are found. In the chromosphere, the lines are mostly formed optically thick, and thus reconnection events in this region are mostly studied only in the lines' wings. However, the Fabry-Pérot spectroscopic imaging instruments commonly used have relatively narrow wavelength coverage and thus do not cover the full spectral extents of the lines. The TR lines in the UV therefore offer excellent opportunities for studying the physical processes of magnetic reconnection and heating in the solar atmosphere. 
The early work on UV bursts has suggested certain common features: they mostly have weak or non-existent signal in the coronal imaging channels of AIA; they generally overlie small-scale, dynamic magnetic features seen in LOS magnetograms from HMI, and emerging flux regions, moving magnetic features and light bridges are the most common locations; and about 10 to $20 \%$ of UV bursts are cospatial with EBs, which are generally interpreted as photospheric reconnection events.

A number of modeling efforts have already been applied to UV bursts with some success (Sect. 5), but one common feature is that it has not been possible to achieve both an EB signature and a UV burst signature from the same event.

Despite the progress already made in describing and understanding UV bursts, we identified some areas for further work with the IRIS data in the preceding text that we summarize here.

- Coordinated studies with Hinode/EIS and IRIS to determine the maximum temperatures reached by the events.

- IRIS imaging and spectroscopy at the maximum possible cadence of 2 seconds to investigate the timescale of intensity fluctuations.

- A wider survey of narrow-line bursts and their connections with the magnetic field.

- A statistical study of bursts in the AIA 1600 and $1700 \AA$ bandpasses during the lifetime of an active region.

- Continued statistical studies of the EB-UV burst connection using $\mathrm{H} \alpha$ data, and the identification of definitive EB signatures in the IRIS chromospheric lines.

- A survey of mini flare ribbons and their properties.

The next solar UV spectroscopic capability for solar physics is the SPICE instrument (Fludra et al. 2013) that will be flown on Solar Orbiter. This will observe two wavelength bands at 704-790 and 973-1049 $\AA$, which give excellent coverage from the chromosphere through the transition region to the corona, and thus will allow the UV burst heating to be tracked through the layers of the atmosphere. The spatial and spectral resolutions will be somewhat lower than those of IRIS, but will be sufficient to see UV bursts.

The Atacama Large Millimeter/submillimeter Array (ALMA; Wedemeyer et al. 2016) promises superb new capability for UV-burst studies when the (far from trivial) technological problems of achieving long-baseline, many-telescope observations including full calibration become solved. Since hydrogen ionizes in UV bursts, their free-free hydrogen opacity becomes very large, even exceeding Lyman- $\alpha$ at high temperature, and since free-free extinction is an LTE process ALMA will directly measure temperatures of UV bursts wherever these are optically thick, at outstanding angular resolution.

DKIST is expected to become operational in late-2019/early-2020 and will resolve structures down to $25 \mathrm{~km}$ size and enable high quality vector magnetograms through higher polarimetric sensitivities compared to other ground-based telescopes. DKIST will have no transition region capability, but the $\mathrm{He}_{\mathrm{I}} \mathrm{D}_{3}$ and $\lambda 10830$ lines may be proxies (Sect. 4.4), and EBs will be prime targets that offer the opportunity to study the reconnection process in unprecedented detail. A very recent study of a UV burst at $75 \mathrm{~km}$ resolution (Smitha et al. 2018) with the Ca II H line from the SUNRISE observatory suggests bursts may have a complex, flare-like structure when observed at ultra-high resolution.

Longer term, a new UV/EUV spectroscopic mission with improved spatial resolution compared to IRIS and a more complete temperature coverage would be ideal for UV bursts. The rapid variability of UV bursts makes sub-second temporal cadence desirable, which is currently limited by the readout times of UV-sensitive CCDs. The recent report ${ }^{4}$ of the Next

\footnotetext{
${ }^{4}$ http://hinode.nao.ac.jp/SOLAR-C/SOLAR-C/Documents/NGSPM_report_170731.pdf.
} 
Generation Solar Physics Mission Science Objectives Team (NGSPM-SOT) recommended a coronal/transition region spectrograph with $0.3^{\prime \prime}$ spatial resolution, and Teriaca et al. (2012) presented an instrument concept called LEMUR (Large European Module for solar Ultraviolet Research) that is compatible with the NGSPM-SOT recommendations. Such an instrument would provide new advances for UV burst studies, particularly when combined with DKIST.

Acknowledgements The authors thank ISSI Bern for support for the team "Solar UV bursts-a new insight to magnetic reconnection". P.R. Young acknowledges funding from NASA grant NNX15AF48G, and thanks the Max Planck Institute in Göttingen for kind hospitality during visits in 2015 and 2016. H. Tian is supported by NSFC grant 41574166, the Max Planck Partner Group program, and the Recruitment Program of Global Experts of China. The work was supported by JSPS KAKENHI Grant Numbers JP16K17671 (PI: S. Toriumi), JP15H05814 (PI: K. Ichimoto), and JP25220703 (PI: S. Tsuneta). G. Vissers acknowledges support under the CHROMOBS grant from the Knut and Alice Wallenberg Foundation. L. Rouppe van der Voort's research is supported by the Research Council of Norway, project number 250810, and through its Centres of Excellence scheme, project number 262622. Z. Huang thanks the support from the NSFC (41404135) and the Young Scholar Program of Shandong University, Weihai (2017WHWLJH07). C.J. Nelson is thankful to the Science and Technology Facilities Council for support received. L.P. Chitta received funding from the European Union's Horizon 2020 research and innovation programme under the Marie Skłodowska-Curie grant agreement No. 707837. A. Berlicki and P. Heinzel acknowledge support by the grant No. 16-18495S of the Czech Funding Agency and by ASI ASCR project RVO: 67985815. We thank L. Kleint for useful discussions. IRIS is a NASA small explorer mission developed and operated by LMSAL with mission operations executed at NASA Ames Research center and major contributions to downlink communications funded by the Norwegian Space Center (NSC, Norway) through an ESA PRODEX contract.

Open Access This article is distributed under the terms of the Creative Commons Attribution 4.0 International License (http://creativecommons.org/licenses/by/4.0/), which permits unrestricted use, distribution, and reproduction in any medium, provided you give appropriate credit to the original author(s) and the source, provide a link to the Creative Commons license, and indicate if changes were made.

\section{Appendix A: Transition Region Imaging}

Imaging the solar atmosphere through narrow bandpass filters enables particular temperature layers to be isolated and studied at high temporal resolution. At visible wavelengths, Fabry-Pérot filters have long been used and they enable high cadence spectral imaging in chromospheric lines such as $\mathrm{H} \alpha$ and $\mathrm{Ca}$ II $\mathrm{H} \& \mathrm{~K}$. For shorter wavelengths $(\approx 100-500 \AA)$ multilayer coatings on optical surfaces enable good reflectivity over a relatively narrow wavelength range (typically $10 \%$ of the central wavelength). If the filters are centered on strong, isolated emission lines then the images can be a good means of isolating a particular temperature regime in the atmosphere. This has been exploited by several space-based instruments with the most advanced being SDO/AIA, which obtains full-disk solar images in seven EUV filters between 94 and $335 \AA$ with around 1 arcsec spatial resolution and a 12 second cadence. The 100-500 A region contains mostly coronal emission lines, with the only strong, isolated cooler lines being He II $\lambda 304$ and Ne VII $\lambda 465$. The former is observed with AIA and is formed around $80 \mathrm{kK}$, however the formation of this line is optically thick, is complex and so the emission not easy to interpret. The Ne VII line is from the upper transition region $(0.5 \mathrm{MK})$, but has not been successfully observed with a multilayer instrument yet.

The lack of filter imaging for the TR means that imaging has been restricted to spectrometers that have the option of a wide slit (usually referred to as a slot) that allows monochromatic imaging in a small spatial region but at the risk of overlapping images from neighboring emission lines. SOHO/CDS had the option of a $90^{\prime \prime} \times 240^{\prime \prime}$ slot that yielded transition 
region images in O v $\lambda 629.7$ although only at 6-10" spatial resolution (e.g., Brković et al. 2000). More recently Hinode/EIS has the options of $40^{\prime \prime}$ and $266^{\prime \prime}$ slots, although there are no strong transition region lines. Useful data have been obtained from the upper TR lines Mg VI $\lambda 269.0$ and Si VII $\lambda 275.4$, however (Ugarte-Urra et al. 2009). Since using a narrow or a wide slit is an either/or option and spectrometer science is typically focused on emission line parameters, then TR imaging has been rare. This has had the consequence that transition region phenomena have generally been classified based on their spectroscopic signature. For example, explosive events (Sect. 3.3) are principally distinguished by their line profiles.

The first filtergraph imager for the transition region is the IRIS slitjaw imager (SJI), which has a filter centered at $1390 \AA$ A that picks up the Si IV lines at 1393.8 and $1402.8 \AA$, as well as the FUV continuum. For intense UV bursts the Si IV emission dominates the signal in the $1400 \AA$ Amages and so the bursts can be confidently assigned to the transition region.

\section{Appendix B: Emission Measure Comparison}

The large Si IV intensities of UV bursts are their most striking feature, and they imply a large emission measure (EM). Here we compare a typical UV burst EM with values derived from the earlier features that seem most similar to them, namely the SMM/UVSP bursts (Sect. 3.4) and active region blinkers (Sect. 3.5).

The expression relating an observed intensity, $I$, to a volume emission measure, $E M_{V}$, for an isothermal plasma of temperature $T$ is

$$
I=G\left(T, N_{\mathrm{e}}\right) \frac{E M_{V}}{A}
$$

where $G\left(T, N_{\mathrm{e}}\right)$ is the contribution function for the observed emission line that is computed using the CHIANTI gofnt IDL software routine. For the lines considered here, $G$ has a weak dependence on density and we compute the function at a density of $10^{12} \mathrm{~cm}^{-3}$ and the temperature at which the function peaks. $G$ contains the element abundance of the emitting ion and we use the "Caffau" solar photospheric abundance set from CHIANTI (see Caffau et al. 2011; Landi et al. 2013). $A$ is the area of the event, and the interpretation varies depending on the spatial resolution of the instrument, as described below.

For IRIS we consider event 1 of Peter et al. (2014)—see also panel (b) of Fig. 2. For computing the $E M_{V}$ value we sum the intensity over a $3 \times 7$ block of spatial pixels centered on the peak intensity value, and we sum across the spectral line profile to obtain the intensity listed in Table 3. The intensity profile across the burst in the $Y$-direction takes a Gaussian shape with a full-width at half-maximum of $0.62^{\prime \prime}$. We thus assume that the event has a spatial size of $0.62^{\prime \prime} \times 0.62^{\prime \prime}$, which we use as $A$ in Eq. 1 .

Hayes and Shine (1987) give Si IV $\lambda 1402.7$ intensities for nine bursts in their Table 1 , and we use the median value for Table 3 . The UVSP pixels for this observation had a size $4^{\prime \prime} \times 4^{\prime \prime}$ and we assume the bursts were fully contained within these pixels, so $A=4 " \times 4^{\prime \prime}$.

For SOHO/CDS we choose the event reported by Young (2004) from the data-set s7616r00 from 1997 April 15, beginning at 21:12 UT. This was a sit-and-stare observation, and the intensity was measured from the exposure with the peak brightness in the $\mathrm{OV}$ $\lambda 629.7$ line. The burst produced a Gaussian distribution of intensity along the CDS slit, with a FWHM consistent with the PSF of the instrument (Young 2004). Therefore $A$ was set to the CDS pixel size, which in this case was $4.00^{\prime \prime} \times 1.68^{\prime \prime}$.

The Hinode/EIS event of Young et al. (2007) was chosen for deriving an emission measure and, to enable direct comparison with CDS, the intensity of O V $\lambda 192.9$ was measured. 
Table 3 Emission measure parameters for a selection of intense transition region brightenings

\begin{tabular}{|c|c|c|c|c|c|c|c|}
\hline Ion & $\begin{array}{l}\text { Wavelength } \\
(\AA)\end{array}$ & $\begin{array}{l}\text { Intensity } \\
\left(\mathrm{erg} \mathrm{cm}^{-2}\right. \\
\left.\mathrm{s}^{-1} \mathrm{sr}^{-1}\right)\end{array}$ & $\begin{array}{l}G\left(\mathrm{erg} \mathrm{cm}^{3}\right. \\
\left.\mathrm{s}^{-1} \mathrm{sr}^{-1}\right)\end{array}$ & $\begin{array}{l}A \\
\left(\mathrm{~cm}^{2}\right)\end{array}$ & $\begin{array}{l}E M_{V} \\
\left(\mathrm{~cm}^{-3}\right)\end{array}$ & Instrument & Reference \\
\hline \multirow[t]{2}{*}{ Si IV } & $\lambda 1402.8$ & $1.86(+6)$ & $1.77(-25)$ & $2.02(+15)$ & $2.12(+46)$ & IRIS & $\begin{array}{l}\text { Peter et al. } \\
(2014)\end{array}$ \\
\hline & & $1.80(+5)$ & $1.77(-25)$ & $8.41(+16)$ & $8.55(+46)$ & SMM/UVSP & $\begin{array}{l}\text { Hayes and } \\
\text { Shine (1987) }\end{array}$ \\
\hline $\mathrm{OV}$ & $\lambda 629.7$ & $5.27(+5)$ & $9.56(-24)$ & $3.53(+16)$ & $1.95(+45)$ & SOHO/CDS & $\begin{array}{l}\text { Young } \\
(2004)\end{array}$ \\
\hline $\mathrm{OV}$ & $\lambda 192.9$ & $8.53(+3)$ & $3.88(-25)$ & $1.05(+6)$ & $2.31(+44)$ & Hinode/EIS & $\begin{array}{l}\text { Young et al. } \\
(2007)\end{array}$ \\
\hline
\end{tabular}

The value of $G$ was calculated by including the two transitions at 192.904 and $192.911 \AA$. As the intensity distribution along the EIS slit had a width consistent with the instrument point spread function, then we considered the event to be unresolved, so the intensity was summed in the $Y$ direction and $A$ was set to $2^{\prime \prime} \times 1^{\prime \prime}$, the pixel size for this observation.

Comparing the EM values in Table 3 we see that the Si IV values are at least an order of magnitude higher than the CDS O v value. We caution that Si IV belongs to the sodium isoelectronic sequence, and there is a well-known problem that ions from the sodium and lithium-like isoelectronic sequences generally yield EM values higher than other species at similar temperatures (Dupree 1972). However, this is unlikely to account for an order of magnitude difference and it suggests a significantly smaller EM in the upper transition region. The EIS event is a further order of magnitude weaker than the CDS event and this, coupled with the apparent lack of intense active region blinkers seen by EIS, suggests that the high-excitation $\lambda 192.9$ line is not as sensitive to the events as the CDS $\lambda 629.7$ line.

Based on the EM, it appears that the SMM/UVSP bursts are consistent with the UV bursts. The most intense of the CDS active region blinkers are weaker than the IRIS and UVSP events, but likely still qualify as UV bursts.

\section{References}

S.E. Alpert, S.K. Tiwari, R.L. Moore, A.R. Winebarger, S.L. Savage, Hi-C observations of sunspot penumbral bright dots. Astrophys. J. 822, 35 (2016). https://doi.org/10.3847/0004-637X/822/1/35

V. Archontis, A.W. Hood, Formation of Ellerman bombs due to 3D flux emergence. Astron. Astrophys. 508, 1469 (2009). https://doi.org/10.1051/0004-6361/200912455

A. Asai, T.T. Ishii, H. Kurokawa, Plasma ejections from a light bridge in a sunspot umbra. Astrophys. J. Lett. 555, L65 (2001). https://doi.org/10.1086/321738

X.Y. Bai, J.T. Su, W.D. Cao, S.Q. Liu, Y.Y. Deng, T.G. Priya, Multi-wavelength observations of a subarcsecond penumbral transient brightening event. Astrophys. J. 823, 60 (2016). https://doi.org/10.3847/0004$637 \mathrm{X} / 823 / 1 / 60$

N. Bello González, S. Danilovic, F. Kneer, On the structure and dynamics of Ellerman bombs. Detailed study of three events and modelling of $\mathrm{H} \alpha$. Astron. Astrophys. 557, A102 (2013). https://doi.org/10.1051/ 0004-6361/201321632

A. Berlicki, P. Heinzel, Observations and NLTE modeling of Ellerman bombs. Astron. Astrophys. 567, A110 (2014). https://doi.org/10.1051/0004-6361/201323244

A. Berlicki, P. Heinzel, E.H. Avrett, Photometric analysis of Ellerman bombs. Mem. Soc. Astron. Ital. 81, 646 (2010)

D. Bewsher, C.E. Parnell, R.A. Harrison, Transition region blinkers I. Quiet-sun properties. Sol. Phys. 206, 21 (2002). https://doi.org/10.1023/A:1014954629349 
S.R. Brannon, Observation and analysis of ballistic downflows in an M-class flare with the interface region imaging spectrograph. Astrophys. J. 833, 101 (2016). https://doi.org/10.3847/1538-4357/833/1/101

P. Brekke, N. Brynildsen, O. Kjeldseth-Moe, P. Maltby, K. Wilhelm, Signatures of magnetic reconnection and observed EUV emission line profiles in an active region. Adv. Space Res. 26, 457 (2000). https:// doi.org/10.1016/S0273-1177(99)01086-8

A. Brković, I. Rüedi, S.K. Solanki, A. Fludra, R.A. Harrison, M.C.E. Huber, J.O. Stenflo, K. Stucki, EUV brightness variations in the quiet Sun. Astron. Astrophys. 353, 1083 (2000)

G.E. Brueckner, J.-D.F. Bartoe, Observations of high-energy jets in the corona above the quiet sun, the heating of the corona, and the acceleration of the solar wind. Astrophys. J. 272, 329 (1983). https://doi.org/10. 1086/161297

G.E. Brueckner, N.P. Patterson, V.E. Scherrer, Spectroscopic far ultraviolet observations of transition zone instabilities and their possible role in a pre-flare energy build-up. Sol. Phys. 47, 127 (1976). https://doi. org/10.1007/BF00152250

G.E. Brueckner, J.-D.F. Bartoe, J.W. Cook, K.P. Dere, D. Socker, H. Kurokawa, M. McCabe, Plasma motions in an emerging flux region. Astrophys. J. 335, 986 (1988). https://doi.org/10.1086/166985

A. Bruzek, On arch-filament systems in spotgroups. Sol. Phys. 2, 451 (1967). https://doi.org/10.1007/ BF00146493

E. Caffau, H.-G. Ludwig, M. Steffen, B. Freytag, P. Bonifacio, Solar chemical abundances determined with a CO5BOLD 3D model atmosphere. Sol. Phys. 268, 255 (2011). https://doi.org/10.1007/s11207-0109541-4

M. Carlsson, R.F. Stein, Formation of solar calcium H and K bright grains. Astrophys. J. 481, 500 (1997). https://doi.org/10.1086/304043

M.C.M. Cheung, M. Rempel, A.M. Title, M. Schüssler, Simulation of the formation of a solar active region. Astrophys. J. 720, 233 (2010). https://doi.org/10.1088/0004-637X/720/1/233

M.C.M. Cheung, B. De Pontieu, T.D. Tarbell, Y. Fu, H. Tian, P. Testa, K.K. Reeves, J. Martínez-Sykora, P. Boerner, J.P. Wülser, J. Lemen, A.M. Title, N. Hurlburt, L. Kleint, C. Kankelborg, S. Jaeggli, L. Golub, S. McKillop, S. Saar, M. Carlsson, V. Hansteen, Homologous helical jets: observations by IRIS, SDO, and Hinode and magnetic modeling with data-driven simulations. Astrophys. J. 801, 83 (2015). https:// doi.org/10.1088/0004-637X/801/2/83

L.P. Chitta, H. Peter, P.R. Young, A closer look at a coronal loop rooted in a sunspot umbra. Astron. Astrophys. 587, A20 (2016). https://doi.org/10.1051/0004-6361/201527340

L.P. Chitta, H. Peter, P.R. Young, Y.-M. Huang, Compact solar UV burst triggered in a magnetic field with a fan-spine topology. Astron. Astrophys. 605, A49 (2017). https://doi.org/10.1051/0004-6361/201730830

S. Danilovic, Simulating Ellerman bomb-like events. Astron. Astrophys. 601, A122 (2017). https://doi.org/ 10.1051/0004-6361/201730403

B. De Pontieu, A.M. Title, J.R. Lemen, G.D. Kushner, D.J. Akin, B. Allard, T. Berger, P. Boerner, M. Cheung, C. Chou, J.F. Drake, D.W. Duncan, S. Freeland, G.F. Heyman, C. Hoffman, N.E. Hurlburt, R.W. Lindgren, D. Mathur, R. Rehse, D. Sabolish, R. Seguin, C.J. Schrijver, T.D. Tarbell, J.-P. Wülser, C.J. Wolfson, C. Yanari, J. Mudge, N. Nguyen-Phuc, R. Timmons, R. van Bezooijen, I. Weingrod, R. Brookner, G. Butcher, B. Dougherty, J. Eder, V. Knagenhjelm, S. Larsen, D. Mansir, L. Phan, P. Boyle, P.N. Cheimets, E.E. DeLuca, L. Golub, R. Gates, E. Hertz, S. McKillop, S. Park, T. Perry, W.A. Podgorski, K. Reeves, S. Saar, P. Testa, H. Tian, M. Weber, C. Dunn, S. Eccles, S.A. Jaeggli, C.C. Kankelborg, K. Mashburn, N. Pust, L. Springer, R. Carvalho, L. Kleint, J. Marmie, E. Mazmanian, T.M.D. Pereira, S. Sawyer, J. Strong, S.P. Worden, M. Carlsson, V.H. Hansteen, J. Leenaarts, M. Wiesmann, J. Aloise, K.-C. Chu, R.I. Bush, P.H. Scherrer, P. Brekke, J. Martinez-Sykora, B.W. Lites, S.W. McIntosh, H. Uitenbroek, T.J. Okamoto, M.A. Gummin, G. Auker, P. Jerram, P. Pool, N. Waltham, The Interface Region Imaging Spectrograph (IRIS). Sol. Phys. 289, 2733 (2014). https://doi.org/10.1007/s11207-014-0485-y

G. Del Zanna, K.P. Dere, P.R. Young, E. Landi, H.E. Mason, CHIANTI-an atomic database for emission lines. Version 8. Astron. Astrophys. 582, A56 (2015). https://doi.org/10.1051/0004-6361/201526827

N. Deng, V. Yurchyshyn, H. Tian, L. Kleint, C. Liu, Y. Xu, H. Wang, Multi-wavelength study of transition region penumbral subarcsecond bright dots using IRIS and NST. Astrophys. J. 829, 103 (2016). https://doi. org/10.3847/0004-637X/829/2/103

K.P. Dere, Solar mass flow in fine-scale structures. Space Sci. Rev. 70, 21 (1994). https://doi.org/10.1007/ BF00777837

K.P. Dere, J.-D.F. Bartoe, G.E. Brueckner, Explosive events in the solar transition zone. Sol. Phys. 123, 41 (1989). https://doi.org/10.1007/BF00150011

K.P. Dere, E. Landi, H.E. Mason, B.C. Monsignori Fossi, P.R. Young, CHIANTI-an atomic database for emission lines. Astron. Astrophys. Suppl. 125, 149 (1997). https://doi.org/10.1051/aas:1997368

J.G. Doyle, A. Giunta, M.S. Madjarska, H. Summers, M. O’Mullane, A. Singh, Diagnosing transient ionization in dynamic events. Astron. Astrophys. 557, L9 (2013). https://doi.org/10.1051/0004-6361/ 201321902 
J. Dudík, G. Del Zanna, E. Dzifčáková, H.E. Mason, L. Golub, Solar transition region lines observed by the interface region imaging spectrograph: diagnostics for the O IV and Si IV lines. Astrophys. J. Lett. 780, L12 (2014). https://doi.org/10.1088/2041-8205/780/1/L12

A.K. Dupree, Analysis of the extreme-ultraviolet quiet solar spectrum. Astrophys. J. 178, 527 (1972). https://doi.org/10.1086/151813

F. Ellerman, Solar hydrogen “bombs”. Astrophys. J. 46, 298 (1917). https://doi.org/10.1086/142366

A.G. Emslie, R.W. Noyes, The characteristics of impulsive solar EUV bursts. Sol. Phys. 57, 373 (1978)

A.G. Emslie, B.R. Dennis, G.D. Holman, H.S. Hudson, Refinements to flare energy estimates: a followup to "energy partition in two solar flare/CME events" by A.G. Emslie et al. J. Geophys. Res. Space Phys. 110, A11103 (2005). https://doi.org/10.1029/2005JA011305.

C. Fang, Q. Hao, M.-D. Ding, Z. Li, Can the temperature of Ellerman bombs be more than 10000 K? Res. Astron. Astrophys. 17, 031 (2017). https://doi.org/10.1088/1674-4527/17/4/31

A. Fludra, D. Griffin, M. Caldwell, P. Eccleston, J. Cornaby, D. Drummond, W. Grainger, P. Greenway, T. Grundy, C. Howe, C. McQuirk, K. Middleton, O. Poyntz-Wright, A. Richards, K. Rogers, C. Sawyer, B. Shaughnessy, S. Sidher, I. Tosh, S. Beardsley, G. Burton, A. Marshall, N. Waltham, S. Woodward, T. Appourchaux, A. Philippon, F. Auchere, E. Buchlin, A. Gabriel, J.-C. Vial, U. Schühle, W. Curdt, D. Innes, S. Meining, H. Peter, S. Solanki, L. Teriaca, M. Gyo, V. Büchel, M. Haberreiter, D. Pfiffner, W. Schmutz, M. Carlsson, S.V. Haugan, J. Davila, P. Jordan, W. Thompson, D. Hassler, B. Walls, C. Deforest, J. Hanley, J. Johnson, P. Phelan, L. Blecha, H. Cottard, G. Paciotti, N. Autissier, Y. Allemand, K. Relecom, G. Munro, A. Butler, R. Klein, A. Gottwald, SPICE EUV spectrometer for the Solar Orbiter mission, in Solar Physics and Space Weather Instrumentation V, 88620F. Proceedings of the SPIE, vol. 8862 (2013). https://doi.org/10.1117/12.2027581.

M.K. Georgoulis, D.M. Rust, P.N. Bernasconi, B. Schmieder, Statistics, morphology, and energetics of Ellerman bombs. Astrophys. J. 575, 506 (2002). https://doi.org/10.1086/341195

M. Grubecka, B. Schmieder, A. Berlicki, P. Heinzel, K. Dalmasse, P. Mein, Height formation of bright points observed by IRIS in Mg II line wings during flux emergence. Astron. Astrophys. 593, A32 (2016). https://doi.org/10.1051/0004-6361/201527358

B.V. Gudiksen, M. Carlsson, V.H. Hansteen, W. Hayek, J. Leenaarts, J. Martínez-Sykora, The stellar atmosphere simulation code Bifrost. Code description and validation. Astron. Astrophys. 531, A154 (2011). https://doi.org/10.1051/0004-6361/201116520

S.L. Guglielmino, L.R. Bellot Rubio, F. Zuccarello, G. Aulanier, S. Vargas Domínguez, S. Kamio, Multiwavelength observations of small-scale reconnection events triggered by magnetic flux emergence in the solar atmosphere. Astrophys. J. 724, 1083 (2010). https://doi.org/10.1088/0004-637X/724/2/1083

G.R. Gupta, D. Tripathi, IRIS and SDO observations of recurrent explosive events. Astrophys. J. 809, 82 (2015). https://doi.org/10.1088/0004-637X/809/1/82

G.R. Gupta, A. Sarkar, D. Tripathi, Observation and modeling of chromospheric evaporation in a coronal loop related to active region transient brightening. Astrophys. J. 857, 137 (2018). https://doi.org/ $10.3847 / 1538-4357 /$ aab95e

V.H. Hansteen, V. Archontis, T.M.D. Pereira, M. Carlsson, L. Rouppe van der Voort, J. Leenaarts, Bombs and flares at the surface and lower atmosphere of the Sun. Astrophys. J. 839, 22 (2017). https://doi.org/ 10.3847/1538-4357/aa6844

R.A. Harrison, EUV blinkers: the significance of variations in the extreme ultraviolet quiet Sun. Sol. Phys. 175, 467 (1997). https://doi.org/10.1023/A:1004964707047

K. Harvey, J. Harvey, Observations of moving magnetic features near sunspots. Sol. Phys. 28, 61 (1973). https://doi.org/10.1007/BF00152912

Y. Hashimoto, R. Kitai, K. Ichimoto, S. Ueno, S. Nagata, T.T. Ishii, M. Hagino, H. Komori, K. Nishida, T. Matsumoto, K. Otsuji, T. Nakamura, T. Kawate, H. Watanabe, K. Shibata, Internal fine structure of Ellerman bombs. Publ. Astron. Soc. Jpn. 62, 879 (2010). https://doi.org/10.1093/pasj/62.4.879

M. Hayes, R.A. Shine, SMM observations of Si IV and O IV bursts in solar active regions. Astrophys. J. 312, 943 (1987). https://doi.org/10.1086/164939

J. Hong, M. Carlsson, M.D. Ding, RADYN simulations of non-thermal and thermal models of Ellerman bombs. Astrophys. J. 845, 144 (2017a). https://doi.org/10.3847/1538-4357/aa80e3

J. Hong, M.D. Ding, W. Cao, Multi-wavelength spectral analysis of Ellerman bombs observed by FISS and IRIS. Astrophys. J. 838, 101 (2017b). https://doi.org/10.3847/1538-4357/aa671e

Z. Hou, Z. Huang, L. Xia, B. Li, M.S. Madjarska, H. Fu, C. Mou, H. Xie, Narrow-line-width UV bursts in the transition region above sunspots observed by IRIS. Astrophys. J. Lett. 829, L30 (2016). https://doi. org/10.3847/2041-8205/829/2/L30

Y. Hou, J. Zhang, T. Li, S. Yang, X. Li, Simultaneous observations of p-mode light walls and magnetic reconnection ejections above sunspot light bridges. Astrophys. J. Lett. 848, L9 (2017). https://doi.org/ 10.3847/2041-8213/aa8edd 
Z. Huang, M.S. Madjarska, L. Xia, J.G. Doyle, K. Galsgaard, H. Fu, Explosive events on a subarcsecond scale in IRIS observations: a case study. Astrophys. J. 797, 88 (2014). https://doi.org/10.1088/0004$637 \mathrm{X} / 797 / 2 / 88$

Z. Huang, L. Xia, B. Li, M.S. Madjarska, Cool transition region loops observed by the interface region imaging spectrograph. Astrophys. J. 810, 46 (2015). https://doi.org/10.1088/0004-637X/810/1/46

Z. Huang, M.S. Madjarska, E.M. Scullion, L.-D. Xia, J.G. Doyle, T. Ray, Explosive events in active region observed by IRIS and SST/CRISP. Mon. Not. R. Astron. Soc. 464, 1753 (2017). https://doi.org/10. 1093/mnras/stw2469

D.E. Innes, P. Brekke, D. Germerott, K. Wilhelm, Bursts of explosive events in the solar network. Sol. Phys. 175, 341 (1997). https://doi.org/10.1023/A:1004997501594

D.E. Innes, L.-J. Guo, Y.-M. Huang, A. Bhattacharjee, IRIS Si IV line profiles: an indication for the plasmoid instability during small-scale magnetic reconnection on the Sun. Astrophys. J. 813, 86 (2015). https:// doi.org/10.1088/0004-637X/813/2/86

H. Isobe, D. Tripathi, V. Archontis, Ellerman bombs and jets associated with resistive flux emergence. Astrophys. J. Lett. 657, L53 (2007). https://doi.org/10.1086/512969

Y. Katsukawa, T.E. Berger, K. Ichimoto, B.W. Lites, S. Nagata, T. Shimizu, R.A. Shine, Y. Suematsu, T.D. Tarbell, A.M. Title, S. Tsuneta, Small-scale jetlike features in penumbral chromospheres. Science 318, 1594 (2007). https://doi.org/10.1126/science.1146046

Y.-H. Kim, V. Yurchyshyn, S.-C. Bong, I.-H. Cho, K.-S. Cho, J. Lee, E.-K. Lim, Y.-D. Park, H. Yang, K. Ahn, P.R. Goode, B.-H. Jang, Simultaneous observation of a hot explosion by NST and IRIS. Astrophys. J. 810, 38 (2015). https://doi.org/10.1088/0004-637X/810/1/38

R. Kitai, On the mass motions and the atmospheric states of moustaches. Sol. Phys. 87, 135 (1983). https:// doi.org/10.1007/BF00151165

E. Landi, P.R. Young, K.P. Dere, G. Del Zanna, H.E. Mason, CHIANTI-an atomic database for emission lines. XIII. Soft X-ray improvements and other changes. Astrophys. J. 763, 86 (2013). https://doi.org/10.1088/0004-637X/763/2/86

K.D. Leka, The vector magnetic fields and thermodynamics of sunspot light bridges: the case for field-free disruptions in sunspots. Astrophys. J. 484, 900 (1997). https://doi.org/10.1086/304363

J.R. Lemen, A.M. Title, D.J. Akin, P.F. Boerner, C. Chou, J.F. Drake, D.W. Duncan, C.G. Edwards, F.M. Friedlaender, G.F. Heyman, N.E. Hurlburt, N.L. Katz, G.D. Kushner, M. Levay, R.W. Lindgren, D.P. Mathur, E.L. McFeaters, S. Mitchell, R.A. Rehse, C.J. Schrijver, L.A. Springer, R.A. Stern, T.D. Tarbell, J.-P. Wuelser, C.J. Wolfson, C. Yanari, J.A. Bookbinder, P.N. Cheimets, D. Caldwell, E.E. Deluca, R. Gates, L. Golub, S. Park, W.A. Podgorski, R.I. Bush, P.H. Scherrer, M.A. Gummin, P. Smith, G. Auker, P. Jerram, P. Pool, R. Soufli, D.L. Windt, S. Beardsley, M. Clapp, J. Lang, N. Waltham, The atmospheric imaging assembly (AIA) on the solar dynamics observatory (SDO). Sol. Phys. 275, 17 (2012). https://doi.org/10.1007/s11207-011-9776-8

Z. Li, C. Fang, Y. Guo, P.-F. Chen, Z. Xu, W.-D. Cao, Diagnostics of Ellerman bombs with high-resolution spectral data. Res. Astron. Astrophys. 15, 1513 (2015). https://doi.org/10.1088/1674-4527/15/9/008

T. Libbrecht, J. Joshi, J.D.L.C. Rodríguez, J. Leenaarts, A.A. Ramos, Observations of Ellerman bomb emission features in $\mathrm{He} \mathrm{I} \mathrm{D}_{3}$ and He I $10830 \AA$ A. Astron. Astrophys. 598, A33 (2017). https://doi.org/10.1051/ 0004-6361/201629266

M.S. Madjarska, Dynamics and plasma properties of an X-ray jet from SUMER, EIS, XRT, and EUVI A \& B simultaneous observations. Astron. Astrophys. 526, A19 (2011). https://doi.org/10.1051/0004-6361/ 201015269

J.T. Mariska, The Solar Transition Region (1992), p. 290

J. Martínez-Sykora, B. De Pontieu, M. Carlsson, V.H. Hansteen, D. Nóbrega-Siverio, B.V. Gudiksen, Twodimensional radiative magnetohydrodynamic simulations of partial ionization in the chromosphere. II. Dynamics and energetics of the low solar atmosphere. Astrophys. J. 847, 36 (2017). https://doi.org/ $10.3847 / 1538-4357 /$ aa8866

C.J. Nelson, S. Shelyag, M. Mathioudakis, J.G. Doyle, M.S. Madjarska, H. Uitenbroek, R. Erdélyi, Ellerman bombs-evidence for magnetic reconnection in the lower solar atmosphere. Astrophys. J. 779, 125 (2013). https://doi.org/10.1088/0004-637X/779/2/125

C.J. Nelson, E.M. Scullion, J.G. Doyle, N. Freij, R. Erdélyi, Small-scale structuring of Ellerman bombs at the solar limb. Astrophys. J. 798, 19 (2015). https://doi.org/10.1088/0004-637X/798/1/19

C.J. Nelson, N. Freij, A. Reid, R. Oliver, M. Mathioudakis, R. Erdélyi, IRIS burst spectra co-spatial to a quietsun Ellerman-like brightening. Astrophys. J. 845, 16 (2017). https://doi.org/10.3847/1538-4357/aa7e7a

L. Ni, B. Kliem, J. Lin, N. Wu, Fast magnetic reconnection in the solar chromosphere mediated by the plasmoid instability. Astrophys. J. 799, 79 (2015). https://doi.org/10.1088/0004-637X/799/1/79

L. Ni, J. Lin, I.I. Roussev, B. Schmieder, Heating mechanisms in the low solar atmosphere through magnetic reconnection in current sheets. Astrophys. J. 832, 195 (2016). https://doi.org/10.3847/0004-637X/832/ 2/195 
L. Ni, V.S. Lukin, N.A. Murphy, J. Lin, Magnetic reconnection in strongly magnetized regions of the low solar chromosphere. Astrophys. J. 852, 95 (2018). https://doi.org/10.3847/1538-4357/aa9edb

D. Nóbrega-Siverio, J. Martínez-Sykora, F. Moreno-Insertis, L. Rouppe van der Voort, Surges and Si IV bursts in the solar atmosphere: understanding IRIS and SST observations through RMHD experiments. Astrophys. J. 850, 153 (2017). https://doi.org/10.3847/1538-4357/aa956c

G. Noci, D. Spadaro, R.A. Zappala, S.K. Antiochos, Mass flows and the ionization states of coronal loops. Astrophys. J. 338, 1131 (1989). https://doi.org/10.1086/167263

K. Olluri, B.V. Gudiksen, V.H. Hansteen, Non-equilibrium ionization effects on the density line ratio diagnostics of O IV. Astrophys. J. 767, 43 (2013). https://doi.org/10.1088/0004-637X/767/1/43

E. Pariat, G. Aulanier, B. Schmieder, M.K. Georgoulis, D.M. Rust, P.N. Bernasconi, Resistive emergence of undulatory flux tubes. Astrophys. J. 614, 1099 (2004). https://doi.org/10.1086/423891

E. Pariat, B. Schmieder, A. Berlicki, Y. Deng, N. Mein, A. López Ariste, S. Wang, Spectrophotometric analysis of Ellerman bombs in the Ca II, H $\alpha$, and UV range. Astron. Astrophys. 473, 279 (2007). https://doi. org/10.1051/0004-6361:20067011

C.E. Parnell, D. Bewsher, R.A. Harrison, Transition-region blinkers-II. Active-region properties. Sol. Phys. 206, 249 (2002). https://doi.org/10.1023/A:1015094119974

H. Peter, H. Tian, W. Curdt, D. Schmit, D. Innes, B. De Pontieu, J. Lemen, A. Title, P. Boerner, N. Hurlburt, T.D. Tarbell, J.P. Wuelser, J. Martínez-Sykora, L. Kleint, L. Golub, S. McKillop, K.K. Reeves, S. Saar, P. Testa, C. Kankelborg, S. Jaeggli, M. Carlsson, V. Hansteen, Hot explosions in the cool atmosphere of the Sun. Science 346, 1255726 (2014). https://doi.org/10.1126/science.1255726

A. Reid, M. Mathioudakis, E. Scullion, J.G. Doyle, S. Shelyag, P. Gallagher, Ellerman bombs with jets: cause and effect. Astrophys. J. 805, 64 (2015). https://doi.org/10.1088/0004-637X/805/1/64

A. Reid, M. Mathioudakis, J.G. Doyle, E. Scullion, C.J. Nelson, V. Henriques, T. Ray, Magnetic flux cancellation in Ellerman bombs. Astrophys. J. 823, 110 (2016). https://doi.org/10.3847/0004-637X/823/2/110

A. Reid, M. Mathioudakis, A. Kowalski, J.G. Doyle, J.C. Allred, Solar Ellerman bombs in 1D radiative hydrodynamics. Astrophys. J. Lett. 835, L37 (2017). https://doi.org/10.3847/2041-8213/835/2/L37

C. Robustini, J. Leenaarts, J. de la Cruz Rodriguez, L. Rouppe van der Voort, Fan-shaped jets above the light bridge of a sunspot driven by reconnection. Astron. Astrophys. 590, A57 (2016). https://doi.org/10.1051/ 0004-6361/201528022

L.H.M. Rouppe van der Voort, R.J. Rutten, G.J.M. Vissers, Reconnection brightenings in the quiet solar photosphere. Astron. Astrophys. 592, A100 (2016). https://doi.org/10.1051/0004-6361/201628889

L. Rouppe van der Voort, B. De Pontieu, G.B. Scharmer, J. de la Cruz Rodríguez, J. Martínez-Sykora, D. Nóbrega-Siverio, L.J. Guo, S. Jafarzadeh, T.M.D. Pereira, V.H. Hansteen, M. Carlsson, G. Vissers, Intermittent reconnection and plasmoids in UV bursts in the low solar atmosphere. Astrophys. J. Lett. 851, L6 (2017). https://doi.org/10.3847/2041-8213/aa99dd

J.R. Roy, The magnetic properties of solar surges. Sol. Phys. 28, 95 (1973). https://doi.org/10.1007/ BF00152915

R.J. Rutten, $\mathrm{H} \alpha$ features with hot onsets. I. Ellerman bombs. Astron. Astrophys. 590, A124 (2016). https:// doi.org/10.1051/0004-6361/201526489

R.J. Rutten, G.J.M. Vissers, L.H.M. Rouppe van der Voort, P. Sütterlin, N. Vitas, Ellerman bombs: fallacies, fads, usage. J. Phys. Conf. Ser. 440(1), 012007 (2013). https://doi.org/10.1088/1742-6596/440/1/ 012007

R.J. Rutten, L.H.M. Rouppe van der Voort, G.J.M. Vissers, Ellerman bombs at high resolution. IV. Visibility in Na I and Mg I. Astrophys. J. 808, 133 (2015). https://doi.org/10.1088/0004-637X/808/2/133

T. Samanta, H. Tian, D. Banerjee, N. Schanche, Dynamics of subarcsecond bright dots in the transition region above sunspots and their relation to penumbral micro-jets. Astrophys. J. Lett. 835, L19 (2017). https:// doi.org/10.3847/2041-8213/835/2/L19

P.H. Scherrer, J. Schou, R.I. Bush, A.G. Kosovichev, R.S. Bogart, J.T. Hoeksema, Y. Liu, T.L. Duvall, J. Zhao, A.M. Title, C.J. Schrijver, T.D. Tarbell, S. Tomczyk, The Helioseismic and Magnetic Imager (HMI) investigation for the Solar Dynamics Observatory (SDO). Sol. Phys. 275, 207 (2012). https://doi. org/10.1007/s11207-011-9834-2

B. Schmieder, P. Mein, J.-C. Vial, E. Tandberg-Hanssen, Dynamics of a surge observed in the C IV and H alpha lines. Astron. Astrophys. 127, 337 (1983)

B. Schmieder, D.M. Rust, M.K. Georgoulis, P. Démoulin, P.N. Bernasconi, Emerging flux and the heating of coronal loops. Astrophys. J. 601, 530 (2004). https://doi.org/10.1086/380199

B. Schmieder, V. Archontis, E. Pariat, in Magnetic Flux Emergence Along the Solar Cycle, ed. by A. Balogh, H. Hudson, K. Petrovay, R. von Steiger (2015), p. 227. https://doi.org/10.1007/978-1-4939-2584-1_8

M. Shimojo, K. Shibata, Physical parameters of solar X-ray jets. Astrophys. J. 542, 1100 (2000). https://doi. org/10.1086/317024 
H.N. Smitha, L.P. Chitta, T. Wiegelmann, S.K. Solanki, Observations of solar chromospheric heating at sub-arcsec spatial resolution. Astron. Astrophys. 617, A128 (2018). https://doi.org/10.1051/0004-6361/ 201833276

S.K. Solanki, Sunspots: an overview. Astron. Astrophys. Rev. 11, 153 (2003). https://doi.org/10.1007/s00159003-0018-4

L. Teriaca, V. Andretta, F. Auchère, C.M. Brown, E. Buchlin, G. Cauzzi, J.L. Culhane, W. Curdt, J.M. Davila, G. Del Zanna, G.A. Doschek, S. Fineschi, A. Fludra, P.T. Gallagher, L. Green, L.K. Harra, S. Imada, D. Innes, B. Kliem, C. Korendyke, J.T. Mariska, V. Martínez-Pillet, S. Parenti, S. Patsourakos, H. Peter, L. Poletto, R.J. Rutten, U. Schühle, M. Siemer, T. Shimizu, H. Socas-Navarro, S.K. Solanki, D. Spadaro, J. Trujillo-Bueno, S. Tsuneta, S.V. Dominguez, J.-C. Vial, R. Walsh, H.P. Warren, T. Wiegelmann, B. Winter, P. Young, LEMUR: large European module for solar ultraviolet research. European contribution to JAXA's Solar-C mission. Exp. Astron. 34, 273 (2012). https://doi.org/10.1007/s10686-011-9274-X

H. Tian, L. Kleint, H. Peter, M. Weber, P. Testa, E. DeLuca, L. Golub, N. Schanche, Observations of subarcsecond bright dots in the transition region above sunspots with the interface region imaging spectrograph. Astrophys. J. Lett. 790, L29 (2014a). https://doi.org/10.1088/2041-8205/790/2/L29

H. Tian, E.E. DeLuca, S.R. Cranmer, B. De Pontieu, H. Peter, J. Martínez-Sykora, L. Golub, S. McKillop, K.K. Reeves, M.P. Miralles, P. McCauley, S. Saar, P. Testa, M. Weber, N. Murphy, J. Lemen, A. Title, P. Boerner, N. Hurlburt, T.D. Tarbell, J.P. Wuelser, L. Kleint, C. Kankelborg, S. Jaeggli, M. Carlsson, V. Hansteen, S.W. McIntosh, Prevalence of small-scale jets from the networks of the solar transition region and chromosphere. Science 346(27), 1255711 (2014b). https://doi.org/10.1126/science.1255711

H. Tian, Z. Xu, J. He, C. Madsen, Are IRIS bombs connected to Ellerman bombs? Astrophys. J. 824, 96 (2016). https://doi.org/10.3847/0004-637X/824/2/96

H. Tian, V. Yurchyshyn, H. Peter, S.K. Solanki, P.R. Young, L. Ni, W. Cao, K. Ji, Y. Zhu, J. Zhang, T. Samanta, Y. Song, J. He, L. Wang, Y. Chen, Frequently occurring reconnection jets from sunspot light bridges. Astrophys. J. 854, 92 (2018a). https://doi.org/10.3847/1538-4357/aaa89d

H. Tian, X. Zhu, H. Peter, J. Zhao, T. Samanta, Y. Chen, Magnetic reconnection at the earliest stage of solar flux emergence. Astrophys. J. 854, 174 (2018b). https://doi.org/10.3847/1538-4357/aaaae6

S.K. Tiwari, R.L. Moore, A.R. Winebarger, S.E. Alpert, Transition-region/coronal signatures and magnetic setting of sunspot penumbral jets: Hinode (SOT/FG), Hi-C, and SDO/AIA observations. Astrophys. J. 816, 92 (2016). https://doi.org/10.3847/0004-637X/816/2/92

S. Toriumi, M.C.M. Cheung, Y. Katsukawa, Light bridge in a developing active region. II. Numerical simulation of flux emergence and light bridge formation. Astrophys. J. 811, 138 (2015a). https://doi.org/10. 1088/0004-637X/811/2/138

S. Toriumi, Y. Katsukawa, M.C.M. Cheung, Light bridge in a developing active region. I. Observation of light bridge and its dynamic activity phenomena. Astrophys. J. 811, 137 (2015b). https://doi.org/10.1088/ 0004-637X/811/2/137

S. Toriumi, Y. Katsukawa, M.C.M. Cheung, Various local heating events in the earliest phase of flux emergence. Astrophys. J. 836, 63 (2017). https://doi.org/10.3847/1538-4357/836/1/63

A. Tortosa-Andreu, F. Moreno-Insertis, Magnetic flux emergence into the solar photosphere and chromosphere. Astron. Astrophys. 507, 949 (2009). https://doi.org/10.1051/0004-6361/200912394

I. Ugarte-Urra, H.P. Warren, D.H. Brooks, Active region transition region loop populations and their relationship to the corona. Astrophys. J. 695, 642 (2009). https://doi.org/10.1088/0004-637X/695/1/642

J.E. Vernazza, E.H. Avrett, R. Loeser, Structure of the solar chromosphere. III-Models of the EUV brightness components of the quiet-sun. Astrophys. J. Suppl. 45, 635 (1981). https://doi.org/10.1086/190731

G.J.M. Vissers, L.H.M. Rouppe van der Voort, R.J. Rutten, Ellerman bombs at high resolution. II. Triggering, visibility, and effect on upper atmosphere. Astrophys. J. 774, 32 (2013). https://doi.org/10.1088/0004$637 \mathrm{X} / 774 / 1 / 32$

G.J.M. Vissers, L.H.M. Rouppe van der Voort, M. Carlsson, Evidence for a transition region response to penumbral microjets in sunspots. Astrophys. J. Lett. 811, L33 (2015). https://doi.org/10.1088/20418205/811/2/L33

G.J.M. Vissers, L.H.M. Rouppe van der Voort, R.J. Rutten, M. Carlsson, B. De Pontieu, Ellerman bombs at high resolution. III. Simultaneous observations with IRIS and SST. Astrophys. J. 812, 11 (2015). https:// doi.org/10.1088/0004-637X/812/1/11

A. Vögler, S. Shelyag, M. Schüssler, F. Cattaneo, T. Emonet, T. Linde, Simulations of magneto-convection in the solar photosphere. Equations, methods, and results of the MURaM code. Astron. Astrophys. 429, 335 (2005). https://doi.org/10.1051/0004-6361:20041507

H. Watanabe, G. Vissers, R. Kitai, L. Rouppe van der Voort, R.J. Rutten, Ellerman bombs at high resolution. I. Morphological evidence for photospheric reconnection. Astrophys. J. 736, 71 (2011). https://doi.org/ 10.1088/0004-637X/736/1/71

S. Wedemeyer, T. Bastian, R. Brajša, H. Hudson, G. Fleishman, M. Loukitcheva, B. Fleck, E.P. Kontar, B. De Pontieu, P. Yagoubov, S.K. Tiwari, R. Soler, J.H. Black, P. Antolin, E. Scullion, S. Gunár, N. 
Labrosse, H.-G. Ludwig, A.O. Benz, S.M. White, P. Hauschildt, J.G. Doyle, V.M. Nakariakov, T. Ayres, P. Heinzel, M. Karlicky, T. Van Doorsselaere, D. Gary, C.E. Alissandrakis, A. Nindos, S.K. Solanki, L. Rouppe van der Voort, M. Shimojo, Y. Kato, T. Zaqarashvili, E. Perez, C.L. Selhorst, M. Barta, Solar science with the Atacama large millimeter/submillimeter array-a new view of our Sun. Space Sci. Rev. 200, 1 (2016). https://doi.org/10.1007/s11214-015-0229-9

K.G. Widing, The extreme-ultraviolet spectrum of a solar impulsive burst. Astrophys. J. 258, 835 (1982). https://doi.org/10.1086/160130

L. Yan, H. Peter, J. He, H. Tian, L. Xia, L. Wang, C. Tu, L. Zhang, F. Chen, K. Barczynski, Self-absorption in the solar transition region. Astrophys. J. 811, 48 (2015). https://doi.org/10.1088/0004-637X/811/1/48

P.R. Young, Intense blinker in active regions, in SOHO 13 Waves, Oscillations and Small-Scale Transients Events in the Solar Atmosphere: Joint View from SOHO and TRACE, ed. by H. Lacoste. ESA Special Publication, vol. 547 (2004), p. 257

P.R. Young, H.E. Mason, The $\mathrm{Mg} / \mathrm{Ne}$ abundance ratio in a recently emerged flux region observed by CDS. Sol. Phys. 175, 523 (1997). https://doi.org/10.1023/A:1004936106427

P.R. Young, G. Del Zanna, H.E. Mason, G.A. Doschek, L. Culhane, H. Hara, Solar transition region features observed with Hinode/EIS. Publ. Astron. Soc. Jpn. 59, 727 (2007)

P.R. Young, K.P. Dere, E. Landi, G. Del Zanna, H.E. Mason, The CHIANTI atomic database. Eur. Phys. J., B, At. Mol. Opt. Phys. 49(7), 074009 (2016). https://doi.org/10.1088/0953-4075/49/7/074009

P.R. Young, F.P. Keenan, R.O. Milligan, H. Peter, A Si IV/O IV electron density diagnostic for the analysis of IRIS solar spectra. Astrophys. J. 857, 5 (2018). https://doi.org/10.3847/1538-4357/aab556

J. Zhao, B. Schmieder, H. Li, E. Pariat, X. Zhu, L. Feng, M. Grubecka, Observational evidence of magnetic reconnection for brightenings and transition region arcades in IRIS observations. Astrophys. J. 836, 52 (2017). https://doi.org/10.3847/1538-4357/836/1/52

X.S. Zhu, H.N. Wang, Z.L. Du, Y.L. Fan, Forced field extrapolation: testing a magnetohydrodynamic (MHD) relaxation method with a flux-rope emergence model. Astrophys. J. 768, 119 (2013). https://doi.org/10. 1088/0004-637X/768/2/119

X. Zhu, H. Wang, Z. Du, H. He, Forced field extrapolation of the magnetic structure of the H $\alpha$ fibrils in the solar chromosphere. Astrophys. J. 826, 51 (2016). https://doi.org/10.3847/0004-637X/826/1/51

H. Zirin, The Solar Atmosphere (1966) 